\title{
Crossings of signed permutations and $q$-Eulerian numbers of type $B$
}

\author{
Sylvie Corteel**, Matthieu Josuat-Vergès*, ${ }^{*}$ And Jang Soo Kim*
}

In this paper, we want to study combinatorics of the type $B$ permutations and in particular, the join statistics crossings, excedances and the number of negative entries. We generalize most of the results known for type $A$ (i.e. zero negative entries) and use a mix of enumerative, algebraic and bijective techniques. This work has been motivated by permutation tableaux of type $B$ introduced by Lam and Williams and natural statistics that can be read on these tableaux. We mostly use (pignose) diagrams and labelled Motzkin paths for the combinatorial interpretations of our results.

\section{Introduction}

Crossings of permutations are a combinatorial statistic with interesting enumerative properties. They appeared in connection with total positivity in Grassmannians (following Postnikov [24] and Williams [31]) and stationary probabilities in a partially asymmetric exclusion process (PASEP) model (following Corteel and Williams [10]), and some tableaux called permutation tableaux [31]. In particular, they gave rise to an interesting $q$-analog of Eulerian numbers [31]. The purpose of this article is to study the type $B$ analogue, jointly with some excedance statistics on signed permutations, and more generally flag excedances. This work is motivated by permutation tableaux of type $B$ introduced by Lam and Williams [21] and natural statistics that can be read on these tableaux [7]. Thus, we obtain $q$-Eulerian numbers of type $B$, or more generally flag $q$-Eulerian numbers of type $B$, and investigate their combinatorial properties.

We show that many previous results can be generalized to this type $B$ case. Firstly, we have a natural symmetry property of the $q$-Eulerian numbers. Whereas there was a rather natural proof in the case of (unsigned)

arXiv: 1203.0154

*All authors are partially supported by the grant ANR08-JCJC-0011.

†Supported by The Austrian Science Foundation (FWF) via the START grant Y463. 
permutations, the proof is much more involved here. We also make a link with another statistic, alignments of permutations. Then, we make use of a method called Matrix Ansatz, as in previous work on (unsigned) permutations and originally related with the PASEP. This has several consequences, in particular we relate our $q$-Eulerian numbers with combinatorics of weighted Motzkin paths (in the style of Flajolet [12] and Françon and Viennot [15]) and give several bijections with signed permutations, that give other combinatorial interpretations of our $q$-Eulerian numbers.

The definitions and results are presented in the next section. The third section is quite short and gives a summary of the statistics on signed permutations. Section 4 is the main section and introduces the $q$-Eulerian numbers of type $B$ and the symmetry property. Section 5 deals with some specific statistics, namely crossings and alignments. Section 6 is on the Matrix Ansatz and different solutions of this Ansatz. Section 7 is a continuation of Section 6 and links our $q$-Eulerian numbers of type $B$ to the combinatorics or labelled Motzkin paths and suffixes. Section 8 gives an enumerative formula for the $q$-Eulerian numbers of type $B$. Most of the times these sections are generalizations of the type $A$ results, but this is not the case for the Section 8 , as we could not take into account the number of negative entries. We then conclude by six open problems.

\section{Definitions and results}

Let $S_{n}$ denote the set of permutations on $[n]=\{1,2, \ldots, n\}$. We will sometimes write a permutation $\pi=\pi_{1} \pi_{2} \cdots \pi_{n}$ as a sequence $\pi=\left(\pi_{1}, \pi_{2}, \ldots, \pi_{n}\right)$. For a permutation $\pi=\pi_{1} \pi_{2} \cdots \pi_{n} \in S_{n}$, a descent of $\pi$ is an integer $i \in[n-1]$ such that $\pi_{i}>\pi_{i+1}$. A weak excedance of $\pi$ is an integer $i \in[n]$ such that $\pi_{i} \geq i$. We denote by $\operatorname{des}(\pi)$ and wex $(\pi)$ the number of descents of $\pi$ and the number of weak excedances of $\pi$ respectively. For positive integers $n$ and $k$, the (type $A$ ) Eulerian number $E_{n, k}$ is the number of $\pi \in S_{n}$ with $\operatorname{des}(\pi)=k-1$. It is well known that $E_{n, k}$ is also equal to the number of $\pi \in S_{n}$ with $\operatorname{wex}(\pi)=k$.

A crossing of a permutation $\pi=\pi_{1} \cdots \pi_{n}$ is a pair $(i, j)$ with $i, j>0$ such that $i<j \leq \pi_{i}<\pi_{j}$ or $i>j>\pi_{i}>\pi_{j}$. We denote by $\operatorname{cr}(\pi)$ the number of crossings of $\pi$.

The $q$-Eulerian number $E_{n, k}(q)$ is defined as follows:

$$
E_{n, k}(q)=\sum_{\substack{\pi \in S_{n} \\ \operatorname{wex}(\pi)=k}} q^{\operatorname{cr}(\pi)}
$$


Crossings of signed permutations and $q$-Eulerian numbers of type $B 193$

Williams [31] showed that $E_{n, k}(-1)=\left(\begin{array}{c}n-1 \\ k-1\end{array}\right)$ and $E_{n, k}(0)=\frac{1}{n}\left(\begin{array}{c}n \\ k\end{array}\right)\left(\begin{array}{c}n \\ k-1\end{array}\right)$ (the Narayana number) and the following symmetry:

$$
E_{n, k}(q)=E_{n, n+1-k}(q) .
$$

We note that Williams [31] first introduced the notion of crossing and alignment of a permutation in the study of totally positive Grassmanian cells. In fact, in [31], Williams defines $E_{n, k}(q)$ using alignments. Corteel [6] showed that there is a simple relation between the number of crossings and the number of alignments, which gives the equivalent definition (1).

Let

$$
A_{n}(y, q)=\sum_{\pi \in S_{n}} y^{\operatorname{wex}(\pi)} q^{\operatorname{cr}(\pi)}=\sum_{k=0}^{n} y^{k} E_{n, k}(q) .
$$

Then $A_{n}(y, q)$ has many interesting meanings related to $q$-Laguerre polynomials, permutations, permutation tableaux, weighted Motzkin paths, and Matrix Ansatz, see [8]. Josuat-Vergès [17] (see also [8]) showed that

$$
A_{n}(y, q)=\sum_{j=0}^{n-k} y^{j}\left(\left(\begin{array}{c}
n \\
j
\end{array}\right)\left(\begin{array}{c}
n \\
j+k
\end{array}\right)-\left(\begin{array}{c}
n \\
j-1
\end{array}\right)\left(\begin{array}{c}
n \\
j+k+1
\end{array}\right)\right) \sum_{i=0}^{k}(-1)^{k} y^{i} q^{i(k+1-i)} .
$$

In this paper, we prove analogous results for signed permutations and type $B$ Eulerian numbers. In general, some permutations statistics are related with the structure of Coxeter group or Weyl group (such as descents and inversions, see [4]) so that it is natural to examine if some particular permutation statistics can be generalized to other groups. Lam and Williams [21], in their work on the totally positive Grassmanian cells, defined analogues of permutation tableaux for other groups. The type $B$ case was studied in $[7,9]$. In particular, the crossings of signed permutations were defined in [7]. In this article, we show how this definition give nice $q$-Eulerian numbers of type $B$, which are a type $B$ analogue of the $E_{n, k}(q)$ mentioned above.

A signed permutation of $[n]$ is a sequence $\pi=\pi_{1} \pi_{2} \cdots \pi_{n}=\left(\pi_{1}, \pi_{2}, \ldots\right.$, $\left.\pi_{n}\right)$ of integers such that $\left(\left|\pi_{1}\right|,\left|\pi_{2}\right|, \ldots,\left|\pi_{n}\right|\right)$ is a permutation of $[n]$ and $\pi_{i} \in[ \pm n]:=\{1,2, \ldots, n,-1,-2, \ldots,-n\}$ for all $i \in[n]$. We denote by $B_{n}$ the set of signed permutations of $[n]$.

A type $B$ descent of $\pi=\pi_{1} \cdots \pi_{n} \in B_{n}$ is an integer $0 \leq i \leq n-1$ satisfying $\pi_{i}>\pi_{i+1}$, where $\pi_{0}=0$. We denote by $\operatorname{des}_{B}(\pi)$ the number of type $B$ descents of $\pi$. Let fwex $(\pi)=2 \operatorname{wex}(\pi)+\operatorname{neg}(\pi)$, where wex $(\pi)=$ $\#\left\{i \in[n]: \pi_{i} \geq i\right\}$ and $\operatorname{neg}(\pi)$ is the number of negative integers in $\pi$.

A crossing of a signed permutation $\pi=\pi_{1} \cdots \pi_{n}$ is a pair $(i, j)$ of positive integers such that 
- $i<j \leq \pi_{i}<\pi_{j}$ or

- $-i<j \leq-\pi_{i}<\pi_{j}$ or

- $i>j>\pi_{i}>\pi_{j}$.

We denote by $\operatorname{cr}(\pi)$ the number of crossings of $\pi$. The notion of crossing of signed permutations was first considered in [7].

Now let $k$ and $n$ be integers with $0 \leq k \leq n$. The type $B$ Eulerian number $E_{n, k}^{B}$ is the number of $\pi \in B_{n}$ with $\operatorname{des}_{B}(\pi)=k$, see [4]. In Section 3, we show that $E_{n, k}^{B}$ is also equal to the number of $\pi \in B_{n}$ with $\lfloor$ fwex $(\pi) / 2\rfloor=k$. For $0 \leq k \leq n$, we define the type $B q$-Eulerian number $E_{n, k}^{B}(q)$ as follows:

$$
E_{n, k}^{B}(q)=\sum_{\substack{\left.\pi \in B_{n} \\ \mathfrak{f w e x}(\pi) / 2\right\rfloor=k}} q^{\operatorname{cr}(\pi)}
$$

Theorem 2.1. For any $0 \leq k \leq n$, we have $E_{n, k}^{B}(-1)=\left(\begin{array}{l}n \\ k\end{array}\right), E_{n, k}^{B}(0)=\left(\begin{array}{l}n \\ k\end{array}\right)^{2}$ (the Narayana numbers of type $B), E_{n, k}(1)$ is the type $B$ Eulerian number, and

$$
E_{n, k}^{B}(q)=E_{n, n-k}^{B}(q)
$$

In Section 5, we consider crossings and alignments of signed permutations and show a simple relation between their numbers, which is an analogous result of Corteel [6].

Let

$$
B_{n}(y, t, q)=\sum_{\pi \in B_{n}} y^{\mathrm{fwex}(\pi)} t^{\operatorname{neg}(\pi)} q^{\operatorname{cr}(\pi)}, \quad B_{n, k}(t, q)=\sum_{\substack{\pi \in B_{n} \\ \mathrm{fwex}(\pi)=k}} t^{\mathrm{neg}(\pi)} q^{\operatorname{cr}(\pi)} .
$$

For example, $B_{0}(y, t, q)=1, B_{1}(y, t, q)=y^{2}+y t$, and $B_{2}(y, t, q)=y^{4}+$ $(2 t+t q) y^{3}+\left(t^{2} q+t^{2}+1\right) y^{2}+t y$. When $t=0$, we have $B_{n}(y, 0, q)=A_{n}(y, q)$, $B_{n, 2 k+1}(0, q)=0$, and $B_{n, 2 k}(0, q)=E_{n, k}(q)$. Note also that

$$
E_{n, k}^{B}(q)=B_{n, 2 k}(1, q)+B_{n, 2 k+1}(1, q) .
$$

Corteel et al. [7] showed that $B_{n}(1, t, q)$ is a generating function for type $B$ permutation tableaux. These tableaux were introduced by Lam and Williams [21]. Their proof works for $B_{n}(y, t, q)$, and we have

$$
B_{n}(y, t, q)=\sum_{T \in \mathcal{P} \mathcal{T}_{B}(n)} y^{2 \operatorname{row}(T)+\operatorname{diag}(T)} t^{\operatorname{diag}(T)} q^{\mathrm{so}(T)},
$$


Crossings of signed permutations and $q$-Eulerian numbers of type $B 195$

where $\operatorname{row}(T)$ is the number of $\operatorname{rows}, \operatorname{diag}(T)$ is the number of ones in the diagonal, and so( $T)$ is the number of superfluous ones, see [7] for the details. Using permutation tableaux Corteel et al. [7] showed the following theorem. Again, they only considered $B_{n}(1, t, q)$, but their proof works for $B_{n}(y, t, q)$.

Theorem 2.2. (Matrix Ansatz [7, Proposition 2]) Let D and E be matrices, $\langle W|$ a row vector, and $|V\rangle$ a column vector, such that:

$$
D E=q E D+D+E, \quad D|V\rangle=|V\rangle, \quad\langle W| E=y t\langle W| D .
$$

Then we have:

$$
B_{n}(y, t, q)=\left\langle W\left|\left(y^{2} D+E\right)^{n}\right| V\right\rangle .
$$

This can be seen as an abstract rule to compute $B_{n}(y, t, q)$, but it is also useful to have explicitly $D, E,\langle W|$ and $|V\rangle$ satisfying the relations (i.e. solutions of the Matrix Ansatz), see [7]. We will give two such solutions in Section 6 .

This Matrix Ansatz and its solutions have several consequences. Firstly, we will show (see Theorem 6.2) a simple recursion for the quantity $B_{n}(y, q)$ involving $q$-derivatives. Secondly, we will show (see Theorem 6.1 ) that the generating function $\sum B_{n}(y, t, q) z^{n}$ has a nice continued fraction expansion. This is the kind of continued fraction (called J-fraction) that are related with moments of orthogonal polynomials [29].

We will show in Section 7 that the two kinds of paths are in bijection with signed permutations, using variants of classical bijections of Françon and Viennot [15], Foata and Zeilberger [14]. We obtain two other interpretations of $B_{n}(y, t, q)$ where $y$ follows a descent statistic and $q$ a pattern statistic. Once again, this is a type $B$ analog of results on permutation tableaux and permutations [11, 28].

At last but not least, we will give (see Theorem 8.1) an exact formula for $B_{n}(y, 1, q)$. It is the analogue of the formula for $B_{n}(y, 0, q)$ in $(3)$ and is obtained from the continued fraction. This will be given in Section 8, and the proof uses techniques developed in [18].

\section{Statistics of signed permutations}

Let $\pi=\pi_{1} \cdots \pi_{n} \in B_{n}$. There are various statistics on signed permutations $[1,4,13]$ :

$$
\begin{aligned}
\operatorname{wex}(\pi) & =\#\left\{i \in[n]: \pi_{i} \geq i\right\}, \\
\operatorname{exc}(\pi) & =\#\left\{i \in[n]: \pi_{i}>i\right\},
\end{aligned}
$$




$$
\begin{aligned}
\operatorname{des}(\pi) & =\#\left\{i \in[n-1]: \pi_{i}>\pi_{i+1}\right\}, \\
\operatorname{des}_{B}(\pi) & =\#\left\{i \in[n-1] \cup\{0\}: \pi_{i}>\pi_{i+1}\right\}, \quad \text { where } \pi_{0}=0, \\
\operatorname{neg}(\pi) & =\#\left\{i \in[n]: \pi_{i}<0\right\}, \\
\operatorname{fwex}(\pi) & =2 \operatorname{wex}(\pi)+\operatorname{neg}(\pi), \\
\operatorname{fexc}(\pi) & =2 \operatorname{exc}(\pi)+\operatorname{neg}(\pi), \\
\operatorname{fdes}(\pi) & =\operatorname{des}(\pi)+\operatorname{des}_{B}(\pi) .
\end{aligned}
$$

It is well known [27, 1.4.3 Proposition] that

$$
\begin{aligned}
\#\left\{\pi \in S_{n}: \operatorname{wex}(\pi)=k\right\} & =\#\left\{\pi \in S_{n}: \operatorname{des}(\pi)=k-1\right\} \\
& =\#\left\{\pi \in S_{n}: \operatorname{exc}(\pi)=k-1\right\} .
\end{aligned}
$$

We will find a similar relation between fwex, fexc, and fdes. We need the following result of Foata and Han.

Lemma 3.1. [13, Section 9] There is a bijection $\psi: B_{n} \rightarrow B_{n}$ such that $\operatorname{fexc}(\pi)=\operatorname{fdes}(\psi(\pi))$.

For $\pi=\pi_{1} \cdots \pi_{n} \in B_{n}$, we define $-\pi \in B_{n}$ by $(-\pi)_{i}=-\left(\pi_{i}\right)$. We also define $\pi^{\mathrm{t}} \in B_{n}$ to be the signed permutation such that $\pi_{i}^{\mathrm{t}}=\epsilon \cdot j$ if and only $\pi_{j}=\epsilon \cdot i$ for $\epsilon \in\{1,-1\}$ and $i, j \in[n]$. In other words, if $M(\pi)$ is the signed permutation matrix of $\pi$, then $M(-\pi)=-M(\pi)$ and $M\left(\pi^{\mathrm{t}}\right)=M(\pi)^{\mathrm{t}}$. Here, the signed permutation matrix $M(\pi)$ is the $n \times n$ matrix whose $(i, j)$-entry is 1 if $\pi_{i}=j,-1$ if $\pi_{i}=-j$, and 0 otherwise. The following lemma is easy to prove.

Lemma 3.2. For $\pi \in B_{n}$, we have

$$
\operatorname{fdes}(\pi)+\operatorname{fdes}(-\pi)=2 n-1, \quad \operatorname{fwex}(\pi)+\operatorname{fexc}\left(\pi^{\mathrm{t}}\right)=2 n \text {. }
$$

The following is a type $B$ analog of (8).

Proposition 3.3. We have

$$
\begin{aligned}
\#\left\{\pi \in B_{n}: \operatorname{fwex}(\pi)=k\right\} & =\#\left\{\pi \in B_{n}: \operatorname{fdes}(\pi)=k-1\right\} \\
& =\#\left\{\pi \in B_{n}: \operatorname{fexc}(\pi)=k-1\right\}
\end{aligned}
$$

and

$$
\#\left\{\pi \in B_{n}: \operatorname{des}_{B}(\pi)=k\right\}=\#\left\{\pi \in B_{n}:\lfloor\operatorname{fwex}(\pi) / 2\rfloor=k\right\}
$$


Crossings of signed permutations and $q$-Eulerian numbers of type $B 197$

Proof. By Lemmas 3.1 and 3.2, we have

$$
\begin{aligned}
\#\left\{\pi \in B_{n}: \operatorname{fwex}(\pi)=k\right\} & =\#\left\{\pi \in B_{n}: \operatorname{fexc}(\pi)=2 n-k\right\} \\
& =\#\left\{\pi \in B_{n}: \operatorname{fdes}(\pi)=2 n-k\right\} \\
& =\#\left\{\pi \in B_{n}: \operatorname{fdes}(\pi)=2 n-1-(2 n-k)\right\} \\
& =\#\left\{\pi \in B_{n}: \operatorname{fdes}(\pi)=k-1\right\} \\
& =\#\left\{\pi \in B_{n}: \operatorname{fexc}(\pi)=k-1\right\} .
\end{aligned}
$$

Equation (11) follows from Equation (10) and the fact that $\operatorname{des}_{B}(\pi)=$ $\lfloor(\operatorname{fdes}(\pi)+1) / 2\rfloor$.

\section{4. $q$-Eulerian numbers of type $B$ and the symmetry property}

The main purpose of this section is to prove (4), which is the symmetry $E_{n, k}^{B}(q)=E_{n, n-k}^{B}(q)$ of type $B$ Eulerian numbers. Observe that by (6) the identity $B_{n, k}(1, q)=B_{n, 2 n+1-k}(1, q)$ implies (4). However, for general $t$, we have $B_{n, k}(t, q) \neq B_{n, 2 n+1-k}(t, q)$. For instance, $B_{1,1}(t, q)=t$ and $B_{1,2}(t, q)=$ 1. There is a way to fix this discrepancy. Let

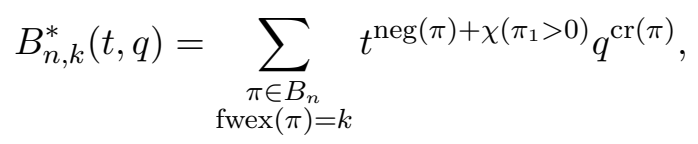

where $\chi\left(\pi_{1}>0\right)$ is 1 if $\pi_{1}>0$ and 0 otherwise. We will prove the following symmetry by a combinatorial argument:

Theorem 4.1. For $1 \leq k \leq 2 n$, we have

$$
B_{n, k}^{*}(t, q)=B_{n, 2 n+1-k}^{*}(t, q) .
$$

In particular, when $t=1$, we have $B_{n, k}(1, q)=B_{n, 2 n+1-k}(1, q)$.

In order to prove Theorem 4.1, we introduce a diagram representing a signed permutation.

\subsection{Pignose diagrams}

Given a set $U$ of $2 n$ distinct integers, an ordered matching on $U$ is a set of ordered pairs $(i, j)$ of integers such that each integer in $U$ appears exactly once. For an ordered matching $M$ on $U$ containing $2 n$ integers $a_{1}<a_{2}<$ $\cdots<a_{2 n}$, we define the standardization $\operatorname{st}(M)$ of $M$ to be the ordered 


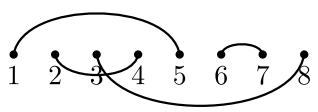

Figure 1: The diagram of the ordered matching $\{(1,5),(4,2),(8,3),(6,7)\}$.

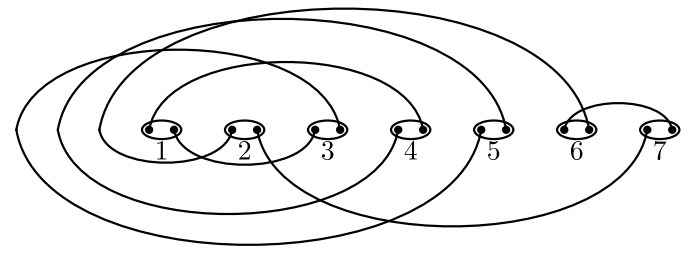

Figure 2: The pignose diagram of $\pi=(4,-6,1,-5,-3,7,2)$.

matching on $[2 n]$ obtained from $M$ by replacing $a_{i}$ with $i$ for each $i \in[2 n]$. For example, if $M=\{(2,6),(5,3),(9,4),(7,8)\}$, then $\operatorname{st}(M)$ is the ordered matching $\{(1,5),(4,2),(8,3),(6,7)\}$ on $[8]$.

We represent an ordered matching $M$ on $U$ as follows. Arrange the integers in $U$ on a horizontal line in increasing order. For each pair $(i, j) \in M$, connect $i$ and $j$ with an upper arc if $i<j$, and with a lower arc if $i>j$, see Figure 1.

A crossing of an ordered matching $M$ is a set of two intersecting arcs, i.e. $\left(i_{1}, j_{1}\right),\left(i_{2}, j_{2}\right)$ satisfying $i_{1}<i_{2}<j_{1}<j_{2}$ or $j_{2}<j_{1}<i_{2}<i_{1}$. We denote by $\operatorname{cr}(M)$ the number of crossings of $M$.

For $\pi=\pi_{1} \cdots \pi_{n} \in B_{n}$, the pignose diagram of $\pi$ is defined as follows. First, we arrange $2 n$ vertices in a horizontal line where the $(2 i-1)$ th vertex and the $2 i$ th vertex are enclosed by an ellipse labeled with $i$ which we call the $i$ th pignose. The left vertex and the right vertex in a pignose are called the first vertex and the second vertex respectively. For each $i \in[n]$, we connect the first vertex of the $i$ th pignose and the second vertex of the $\pi_{i}$ th pignose with an arc in the following way. If $\pi_{i}>0$, then draw an arc above the horizontal line if $\pi_{i} \geq i$ and below the horizontal line if $\pi_{i}<i$. If $\pi_{i}<0$, then we draw an arc starting from the first vertex of the $i$ th pignose below the horizontal line to the second vertex of the $\pi_{i}$ th pignose above the horizontal line like a spiral oriented clockwise. We draw these spiral arcs so that these are not crossing each other below the horizontal line. See Figure 2. We note that essentially the notion of the pignose diagram for permutations was first considered in [23].

For $\pi \in B_{n}$, one can easily check that

- $\operatorname{cr}(\pi)$ is the number of unordered pairs of two arcs crossing each other in the pignose diagram of $\pi$, 
Crossings of signed permutations and $q$-Eulerian numbers of type $B 199$

- $\mathrm{fwex}(\pi)$ is twice the number of upper arcs plus the number of spiral arcs, or equivalently, the number of vertices with a half arc above the horizontal line,

- $\operatorname{neg}(\pi)$ is the number of spiral arcs.

Since $S_{n}$ is contained in $B_{n}$, the pignose diagram for $\pi \in S_{n}$ is also defined. Note that the pignose diagram of $\pi \in S_{n}$ can be considered as an ordered matching on $[2 n]$ by removing the ellipses enclosing two vertices and labeling the $2 n$ vertices with $1,2 \ldots, 2 n$ from left to right. We call an ordered matching that can be obtained in this way a pignose matching. Not all ordered matchings are pignose matchings. In order to determine whether a given ordered matching is a pignose matching, we need some definitions.

For an ordered matching $M$ on $[2 n]$ and an integer $i \in[2 n]$ we say that

- $i$ has a half arc of type $\checkmark$ if $i$ is the left vertex of an upper arc in the diagram of $M$.

- $i$ has a half arc of type $\boldsymbol{\rho}$ if $i$ is the right vertex of a lower arc in the diagram of $M$.

- $i$ has a half arc of type $\boldsymbol{\succ}$ if $i$ is the right vertex of an upper arc in the diagram of $M$.

- $i$ has a half arc of type $\boldsymbol{a}$ if $i$ is the left vertex of a lower arc in the diagram of $M$.

One can easily prove the following proposition.

Proposition 4.2. An ordered matching $M$ on $[2 n]$ is a pignose matching if and only if in the representation of $M$ each odd integer has a half arc of type $\boldsymbol{\checkmark}$ or $\boldsymbol{\bullet}$, and each even integer has a half arc of type $\boldsymbol{\bullet}$ or $\boldsymbol{\vee}$.

The following lemma was first observed by de Médicis and Viennot [23, Lemme 3.1 Cas b.4]. For the readers' convenience, we include a proof as well.

Lemma 4.3. For $\pi \in S_{n}$ and an integer $k \in[n]$, the number of integers $i \in[n]$ with $i \leq k \leq \pi_{i}$ is equal to the number of integers $i \in[n]$ with $\pi_{i}<k<i$ plus 1. See Figure 3 for the pictorial meaning.

Proof. Consider the pignose diagram of $\pi$ with a vertical line dividing the pignose of $k$ in the middle. Let $x$ (resp. $y$ ) be the number of lower (resp. upper) arcs intersecting with the vertical line. Let $a, b, c, d$ be, respectively, the number of half arcs to the left of the vertical line of types $\boldsymbol{\bullet}, \boldsymbol{c}, \boldsymbol{v}$ and 9.

For each pignose, the first vertex has a half edge of the first or the second type and the second vertex has a half edge of the third or the fourth type. Thus, $a+b=c+d+1$. Since $x=a-c$ and $y=d-b$, we are done. 


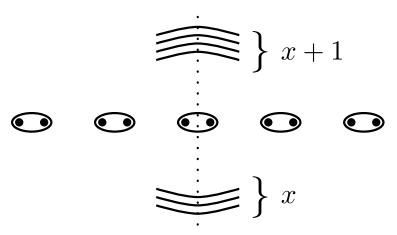

Figure 3: In the pignose diagram of $\pi \in S_{n}$, if we draw a vertical line dividing the $k$ th pignose in the middle, then the number of upper arcs intersecting with this line is equal to the number of lower arcs intersecting with this line plus 1.

\subsection{Proof of Theorem 4.1}

Let

$$
\begin{aligned}
B_{n}^{+} & =\left\{\pi \in B_{n}: \pi_{1}>0\right\}, \quad B_{n}^{-}=\left\{\pi \in B_{n}: \pi_{1}<0\right\}, \\
B_{n, k}^{+}(t, q) & =\sum_{\substack{\pi \in B_{n}^{+} \\
f \operatorname{wex}(\pi)=k}} t^{\mathrm{neg}(\pi)} q^{\operatorname{cr}(\pi)}, \quad B_{n, k}^{-}(t, q)=\sum_{\substack{\pi \in B_{n}^{-} \\
f \operatorname{wex}(\pi)=k}} t^{\mathrm{neg}(\pi)} q^{\operatorname{cr}(\pi)} .
\end{aligned}
$$

Then

$$
B_{n, k}^{*}(t, q)=t B_{n, k}^{+}(t, q)+B_{n, k}^{-}(t, q) .
$$

In this subsection, we will prove the following proposition.

Proposition 4.4. There is a bijection $\phi: B_{n}^{+} \rightarrow B_{n}^{-}$such that

$$
\begin{array}{r}
\operatorname{cr}(\phi(\pi))=\operatorname{cr}(\pi), \quad \operatorname{neg}(\phi(\pi))=\operatorname{neg}(\pi)+1, \\
\quad \operatorname{fwex}(\phi(\pi))=2 n+1-\operatorname{fwex}(\pi) .
\end{array}
$$

Thus,

$$
t B_{n, k}^{+}(t, q)=B_{n, 2 n+1-k}^{-}(t, q), \quad B_{n, k}^{-}(t, q)=t B_{n, 2 n+1-k}^{+}(t, q) .
$$

Note that Theorem 4.1 follows from (12) and (14). In order to prove Proposition 4.4, we need some definitions and lemmas.

Given an ordered matching $M$ on $[2 n]$, we define $\rho(M)$ to be the ordered matching $\operatorname{st}\left(M^{\prime}\right)$ where $M^{\prime}$ is the ordered matching on $\{2,3, \ldots, 2 n+1\}$ obtained from $M$ by replacing 1 with $2 n+1$. In other words, $\rho(M)$ is obtained from $M$ by moving the first vertex to the end and reflecting the arc adjacent to this vertex, see Figure 4. 
Crossings of signed permutations and $q$-Eulerian numbers of type $B 201$

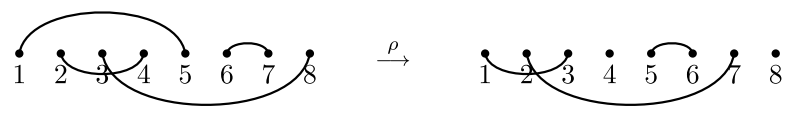

Figure 4: If $M=\{(1,5),(4,2),(8,3),(6,7)\}$ (on the left), then $M^{\prime}=$ $\{(9,5),(4,2),(8,3),(6,7)\}$ and $\rho(M)=\operatorname{st}\left(M^{\prime}\right)=\{(8,4),(3,1),(7,2),(5,6)\}$ (on the right).

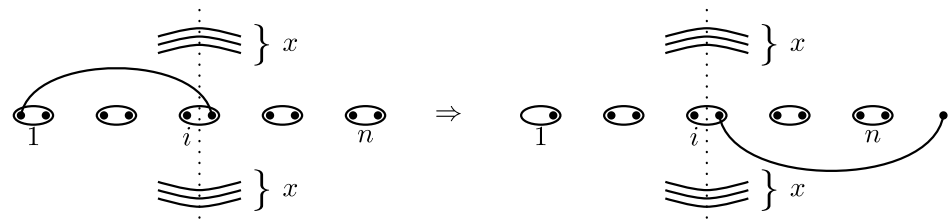

Figure 5: The change of crossings from $M$ to $\rho(M)$.

We denote $\rho^{(k)}=\overbrace{\rho \circ \cdots \circ \rho}^{k}$.

Lemma 4.5. Let $M$ be a pignose matching on $[2 n]$. Then $\rho^{(k)}(M)$ has the same number of crossings as $M$ for all positive integers $k$.

Proof. Note that $\rho^{(2)}(M)$ is a pignose matching. Thus, it suffices to prove for $k=1$ and $k=2$.

Considering $M$ as a pignose diagram of a permutation in $S_{n}$, assume that 1 is connected to $i$. By Lemma 4.3, if we draw a vertical line between the two vertices in the $i$ th pignose, the number, say $x$, of upper arcs above $i$ except $(1, i)$ is equal to the number of lower arcs below $i$. Therefore, when we go from $M$ to $\rho(M)$, we lose $x$ crossings and obtain new $x$ crossings as shown in Figure 5.

This proves the assertion for $k=1$.

To prove for $k=2$ we define the following. Given an ordered matching $N$, let $\bar{N}$ be the ordered matching obtained by reflecting $N$ along the horizontal line. It is easy to see that $\operatorname{cr}(N)=\operatorname{cr}(\bar{N})$ and $\overline{\rho(N)}=\rho(\bar{N})$. Moreover if $N$ is a pignose matching then so is $\overline{\rho(N)}$. Thus, we have

$$
\operatorname{cr}\left(\rho^{(2)}(M)\right)=\operatorname{cr}\left(\overline{\rho^{(2)}(M)}\right)=\operatorname{cr}(\rho(\overline{\rho(M)})) .
$$

Since $\overline{\rho(M)}$ is a pignose matching, using the assertion for $k=1$, we obtain that $(15)$ is equal to 


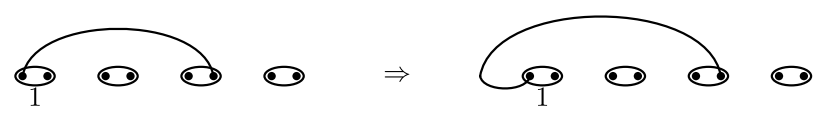

Figure 6: The map $\pi \mapsto \pi^{-}$simply changes the upper arc adjacent to 1 to a spiral arc.

$$
\operatorname{cr}(\overline{\rho(M)})=\operatorname{cr}(\rho(M))=\operatorname{cr}(M)
$$

Therefore, $\operatorname{cr}\left(\rho^{(2)}(M)\right)=\operatorname{cr}(M)$, and we are done.

For an ordered matching $M$ on $[2 n]$ we define $M^{\mathbf{r}}$ to be the ordered matching obtained from $M$ by replacing $i$ with $2 n+1-i$ for each $i \in[2 n]$. Pictorially, $M^{\mathbf{r}}$ is obtained from $M$ by taking a $180^{\circ}$ rotation.

Lemma 4.6. Let $M$ be a pignose matching on $[2 n]$. Then $N=\left(\rho^{(2 k+1)}(M)\right)^{\mathbf{r}}$ is also a pignose matching and $\operatorname{cr}(M)=\operatorname{cr}(N)$.

Proof. Note that $\rho^{(2)}(M)$ is a pignose matching. Thus it suffices to prove that $(\rho(M))^{\mathbf{r}}$ is a pignose matching, which easily follows from Proposition 4.2.

For $\pi \in B_{n}$, let $\pi^{-}=\left(-\pi_{1}\right) \pi_{2} \cdots \pi_{n}$.

Lemma 4.7. The map $\pi \mapsto \pi^{-}$is a bijection from $B_{n}^{+}$to $B_{n}^{-}$. Moreover, we have $\operatorname{cr}(\pi)=\operatorname{cr}\left(\pi^{-}\right), \operatorname{neg}(\pi)=\operatorname{neg}\left(\pi^{-}\right)-1$, and fwex $(\pi)=\operatorname{fwex}\left(\pi^{-}\right)+1$.

Proof. This is an immediate consequence of the following observation: if $\pi \in B_{n}^{+}$, the pignose diagram of $\pi^{-}$is obtained from the pignose diagram of $\pi$ by changing the upper arc adjacent to 1 to a spiral arc as shown in Figure 6.

Now we are ready to define the map $\phi: B_{n}^{+} \rightarrow B_{n}^{-}$in Proposition 4.4. Suppose $\pi \in B_{n}^{+}$and neg $(\pi)=m$. We make the pignose diagram of $\pi$ to be a pignose matching on $[2 m+2 n]$ by dividing each spiral arc into one upper arc and one lower arc so that the left endpoint of the upper arc is to the left of the left endpoint of the lower arc, see Figure 7.

Let $M$ be the pignose matching obtained in this way, and let $N=$ $\left(\rho^{(2 m+1)}(M)\right)^{\mathbf{r}}$. By Lemma $4.6, N$ is also a pignose matching on $[2 m+2 n]$ and $\operatorname{cr}(M)=\operatorname{cr}(N)$. It is straightforward to check that $N$ satisfies the following properties.

1. For each $i \in[2 m]$, the $i$ th vertex is connected to the $j$ th vertex for some $j>2 m$.

2. The first $m$ lower arcs do not cross each other.

3 . The $(2 m+1)$ st vertex has an upper half arcs. 
Crossings of signed permutations and $q$-Eulerian numbers of type $B 203$

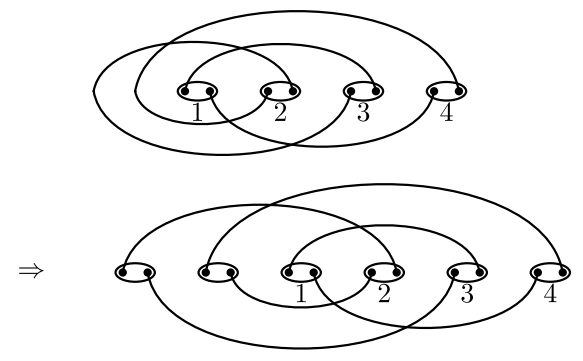

Figure 7: Chaning the signed permutation $\pi=(3,-4,-2,1) \in B_{4}^{+}$with $\operatorname{neg}(\pi)=2$ to the signed permutation in $B_{6}$.

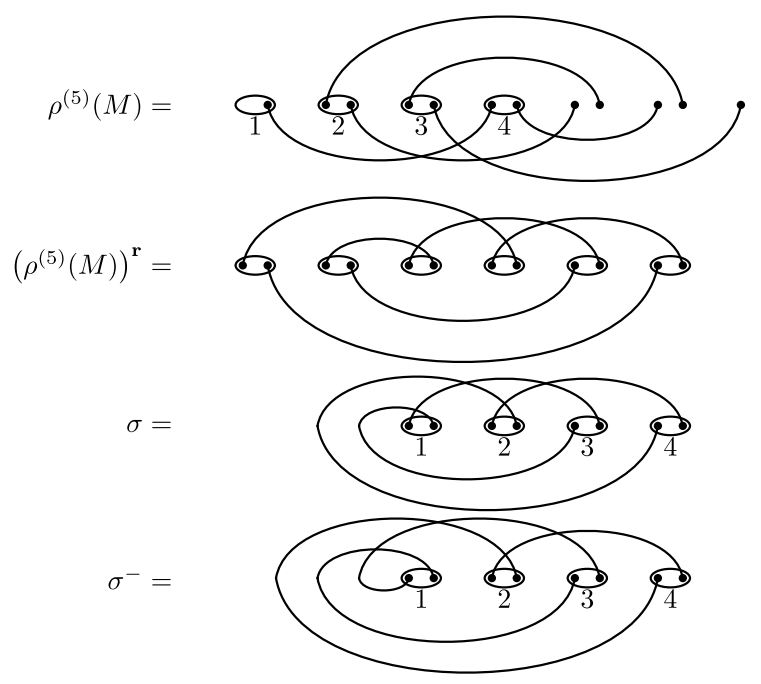

Figure 8: Computing $\rho^{(5)}(M),\left(\rho^{(5)}(M)\right)^{\mathbf{r}}, \sigma$, and $\sigma^{-}$, where $\pi=$ $(3,-4,-2,1) \in B_{4}^{+}$and $M$ is the diagram on the right in Figure 7.

4. The number of upper half arcs adjacent to the last $2 n$ vertices is $2 n+$ $2-k$.

By the first and the second properties, we can make $N$ to be the pignose diagram of a signed permutation, say $\sigma \in B_{n}$, by identifying the $(2 i-1)$ th vertex and the $(2 i)$ th vertex for each $i \in[m]$. Then $\operatorname{neg}(\pi)=\operatorname{neg}(\sigma)$. By the third property, we have $\sigma \in B_{n}^{+}$, and by the fourth property, we have fwex $(\sigma)=2 n+2-k$. We define $\phi(\pi)$ to be $\sigma^{-}$. Clearly, $\phi$ is a bijection from $B_{n}^{+}$to $B_{n}^{-}$. By Lemma 4.7, $\phi$ satisfies (13). This finishes the proof of Proposition 4.4. See Figure 8 for an example. 


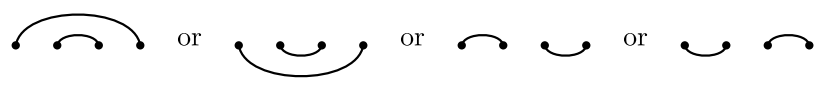

Figure 9: An alignment is an unordered pair of arcs which look like one of the four configurations.

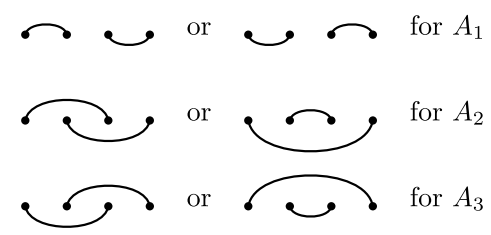

Figure 10: The relative locations of a pair of arcs in $A_{1}, A_{2}$ and $A_{3}$.

\section{Crossings and alignments}

For a permutation $\sigma \in S_{n}$, an alignment is a pair $(i, j)$ of integers $i, j \in[n]$ satifying one of the following: $i<j<\pi_{j}<\pi_{i}, \pi_{i}<\pi_{j}<j<i, i<\pi_{i}<$ $\pi_{j}<j$, and $\pi_{i}<i<j<\pi_{j}$, see Figure 9 .

Let $\operatorname{al}(\sigma)$ denote the number of alignments of $\sigma$. The following proposition was first proved by the first author [6] using rather technical calculations. Here we provide another proof which is more combinatorial.

Proposition 5.1. If $\sigma \in S_{n}$ has $k$ weak excedances, then

$$
\operatorname{cr}(\sigma)+\operatorname{al}(\sigma)=(k-1)(n-k) .
$$

Proof. Since wex $(\pi)=k$, we have $k$ upper arcs and $n-k$ lower arcs in the pignose diagram of $\pi$.

Let $A$ be the set of pairs $(U, L)$ of an upper arc $U$ and a lower arc $L$. Then there are $k(n-k)$ elements in $A$. We define the subsets $A_{1}, A_{2}$ and $A_{3}$ of $A$ as follows: for $U=\left(i, \pi_{i}\right)$ and $L=\left(j, \pi_{j}\right)$ we have

- $(U, L) \in A_{1}$ if and only if $i<\pi_{i}<\pi_{j}<j$ or $\pi_{j}<j<i<\pi_{i}$,

- $(U, L) \in A_{2}$ if and only if $i<\pi_{j}<\pi_{i}<j$ or $\pi_{j}<i<\pi_{i}<j$,

- $(U, L) \in A_{3}$ if and only if $\pi_{j}<i<j<\pi_{i}$ or $i<\pi_{j}<j<\pi_{i}$.

See Figure 10.

Observe that $A=A_{1} \uplus A_{2} \uplus A_{3}$. Fix an upper arc $U=\left(i, \pi_{i}\right)$. Note that the right endpoint of $U$ is the second vertex of the $\pi_{i}$ th pignose. Then by Lemma 4.3 , the number of elements $(U, L) \in A_{2}$ is equal to the number of 
Crossings of signed permutations and $q$-Eulerian numbers of type $B 205$

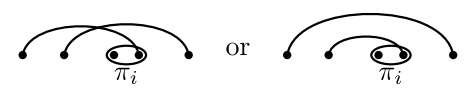

Figure 11: The relative locations of $U=\left(i, \pi_{i}\right)$ and $U^{\prime}=\left(j, \pi_{j}\right)$. Here $U$ is the arc whose right endpoint is the second vertex of the $\pi_{i}$ th pignose and $U^{\prime}$ is the other arc.

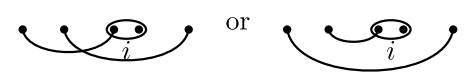

Figure 12: The relative locations of $L=\left(i, \pi_{i}\right)$ and $L^{\prime}=\left(j, \pi_{j}\right)$. Here $L$ is the arc whose right endpoint is the first vertex of the $i$ th pignose and $L^{\prime}$ is the other arc.

pairs $\left(U, U^{\prime}\right)$ of upper arcs where $U^{\prime}=\left(j, \pi_{j}\right)$ satisfying $i<j<\pi_{i}<\pi_{j}$ or $j<i \leq \pi_{i}<\pi_{j}$, see Figure 11 .

Now fix a lower arc $L=\left(i, \pi_{i}\right)$. Note that the right endpoint of $L$ is the first vertex of the $i$ th pignose. Again by Lemma 4.3, the number of elements $(U, L) \in A_{3}$ is one more than the number of pairs $\left(L, L^{\prime}\right)$ of lower arcs where $L^{\prime}=\left(j, \pi_{j}\right)$ satisfying $\pi_{i}<\pi_{j}<i<j$ or $\pi_{j}<\pi_{i}<i<j$, see Figure 12.

Observe that a crossing or an alignment is either an element in $A_{1}$ or a pair of arcs as shown in Figures 11 and 12. Since we have $n-k$ lower arcs, the number of pairs of arcs in Figure 12 is $\left|A_{3}\right|-(n-k)$. Thus, the total number of crossings and alignments is equal to

$$
\left|A_{1}\right|+\left|A_{2}\right|+\left(\left|A_{3}\right|-(n-k)\right)=k(n-k)-(n-k)=(k-1)(n-k) .
$$

Now we define another representation of a signed permutation. Note that a signed permutation $\pi=\pi_{1} \cdots \pi_{n} \in B_{n}$ can be considered as a bijection on $[ \pm n]=\{1,2, \ldots, n,-1,-2, \ldots,-n\}$ with $\pi(i)=\pi_{i}$ and $\pi(-i)=-\pi_{i}$ for $i \in[n]$.

We define the full pignose diagram of $\pi \in B_{n}$ as follows. First we arrange $2 n$ pignoses in a horizontal line which are labeled with $-n,-(n-$ $1), \ldots,-1,1,2, \ldots, n$. The pignose labeled by $i$ is called positive if $i>0$, and negative otherwise. The first vertex and the second vertex of a positive pignose are, respectively, the left vertex and the right vertex of the pignose. The first vertex and the second vertex of a negative pignose are, respectively, the right vertex and the left vertex of the pignose. For each $i \in[ \pm n]$, we connect the first vertex of the pignose labeled with $i$ and the second vertex of the pignose labeled with $\pi(i)$ with an arc in the following way. If $\pi_{i}>0$, 


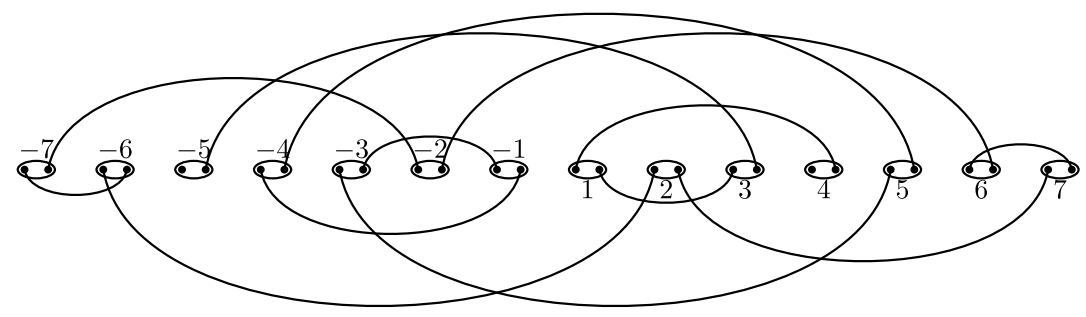

Figure 13: The full pignose diagram of $\pi=[4,-6,1,-5,-3,7,2]$.
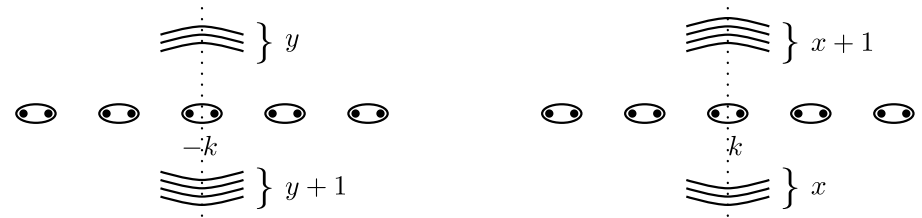

Figure 14: The number of upper and lower arcs crossing a vertical line dividing a pignose in a full pignose diagram.

draw an arc above the horizontal line if $\pi(i) \geq i$, and below the horizontal line if $\pi(i)<i$. See Figure 13 for an example.

The following lemma can be shown by the same argument in the proof of Lemma 4.3.

Lemma 5.2. Let $\pi \in B_{n}$ and $k \in[n]$. In the full pignose diagram of $\pi$ if we draw a vertical line dividing the pignose labeled $-k$ (resp. $k$ ) in the middle, then the number of upper arcs intersecting with this line is equal to the number of lower arcs intersecting with this line minus 1 (resp. plus 1) as shown in Figure 14.

We define alignments of a signed permutation similarly. An alignment of $\pi \in B_{n}$ is a pair $(i, j)$ of integers $i, j \in[ \pm n]$ satifying one of the following: $i<j<\pi(j)<\pi(i), \pi(i)<\pi(j)<j<i, i<\pi(i)<\pi(j)<j$, and $\pi(i)<i<j<\pi(j)$. In other words, an alignment of $\pi \in B_{n}$ is an unordered pair of arcs in the full pignose diagram of $\pi$ whose relative locations are as shown in Figure 9 . We denote by al $(\pi)$ the number of alignments of $\pi$.

Note that the number of two arcs intersecting with each other in the full pignose diagram of $\pi \in B_{n}$ is equal to $2 \operatorname{cr}(\pi)$.

Now we can prove a type $B$ analog of Proposition 5.1.

Proposition 5.3. For $\pi \in B_{n}$ with $\operatorname{fwex}(\pi)=k$, we have

$$
2 \operatorname{cr}(\pi)+\operatorname{al}(\pi)=n^{2}-2 n+k .
$$


Proof. Since the number of positive upper half arcs is equal to fwex $(\pi)=k$, the number of positive lower half arcs is equal to $2 n-k$.

Let $A$ be the set of pairs $(U, L)$ of an upper $\operatorname{arc} U$ and a lower $\operatorname{arc} L$. Since we have $n$ upper arcs and $n$ lower arcs in total, there are $n^{2}$ elements in $A$. Using Lemma 5.2 and the same argument as in the proof of Proposition 5.1, one can easily see that

$$
2 \operatorname{cr}(\pi)+\operatorname{al}(\pi)=n^{2}-a-b,
$$

where $a$ is the number of upper arcs whose right endpoint is the first vertex of a negative pignose, and $b$ is the number of lower arcs whose right endpoint is the first vertex of a positive pignose. By the symmetry of the full pignose diagram, $a$ is equal to the number of lower arcs whose left endpoint is the second vertex of a positive pignose. Thus, $a+b$ is the number of positive half arcs, which is $2 n-k$, and we obtain the desired formula.

\section{Two solutions of the Matrix Ansatz}

If some matrices $D, E$, a row vector $\langle W|$ and a column vector $|V\rangle$ are given explicitly, it is straightforward to check whether they satisfy the Matrix Ansatz:

$$
D E=q E D+D+E, \quad D|V\rangle=|V\rangle, \quad\langle W| E=y t\langle W| D .
$$

But it is not obvious how to find such matrices and vectors. We provide two solutions in the form of semi-infinite tridiagonal matrices, i.e. the index set is $\mathbb{N}$ and $D=\left(D_{i, j}\right)_{i, j \in \mathbb{N}}, E=\left(E_{i, j}\right)_{i, j \in \mathbb{N}}$ are such that $D_{i, j}=E_{i, j}=0$ if $|j-i|>1$.

They can be obtained using the following observation: if $X$ and $Y$ are matrices such that $X Y-q Y X=I$ (where $I$ is the identity), then $D=$ $X(I+Y)$ and $E=Y X(I+Y)$ satisfy

$$
\begin{aligned}
D E-q E D & =X(I+Y) Y X(I+Y)-q Y X(I+Y) X(I+Y) \\
& =(X Y+X Y Y-q Y X-q Y X Y) X(I+Y) \\
& =(I+Y) X(I+Y)=D+E .
\end{aligned}
$$

Then we can look for $\langle W|$ (respectively, $|V\rangle$ ) as a left (respectively, right) eigenvector of $y t D-E$ (respectively, $D)$. 


\subsection{Solution 1}

Let $X=\left(X_{i, j}\right)_{i, j \geq 0}$ and $Y=\left(Y_{i, j}\right)_{i, j \geq 0}$ where

$$
\begin{array}{r}
X_{i, i+1}=[i+1]_{q} \text { and } X_{i, j}=0 \text { otherwise, } \\
Y_{i+1, i}=1, Y_{i, i}=t y q^{i} \text { and } Y_{i, j}=0 \text { otherwise, }
\end{array}
$$

and

$$
\langle W|=(1,0,0, \ldots), \quad| V\rangle=(1,0,0, \ldots)^{\mathrm{t}} .
$$

We can check that $X Y-q Y X=I$ and that $D=X(I+Y)$ and $E=Y X(I+$ $Y$ ) together with $\langle W|$ and $|V\rangle$ provide a solution of (7). The coefficients are:

$$
D_{i, i}=[i+1]_{q}, \quad D_{i, i+1}=\left(1+y t q^{i+1}\right)[i+1]_{q}, \quad D_{i, j}=0 \text { otherwise }
$$

and

$$
\begin{array}{cl}
E_{i, i}=[i]_{q}+y t q^{i}\left([i]_{q}+[i+1]_{q}\right), & E_{i, i+1}=y t q^{i}\left(1+y t q^{i+1}\right)[i+1]_{q} \\
E_{i+1, i}=[i+1]_{q}, & E_{i, j}=0 \text { otherwise. }
\end{array}
$$

Note that $\langle W|$ and $|V\rangle$ are such that the quantity

$$
B_{n}(y, t, q)=\left\langle W\left|\left(y^{2} D+E\right)^{n}\right| V\right\rangle .
$$

is the coefficient $\left(\left(y^{2} D+E\right)^{n}\right)_{0,0}$ of the matrix $\left(y^{2} D+E\right)^{n}$. It is possible to see $y^{2} D+E$ as a transfer matrix, see [27, Section 4.7]. This is a general method used in the enumeration of "walks", i.e. finite sequences of vertices in a graph where two successive elements are related by an edge, see loc. cit. for details. This method shows that $B_{n}(y, t, q)$ is the weighted generating function of walks in $\mathbb{N}$ of $n$ steps, starting at 0 , ending at 0 , and each step being either $i \rightarrow i+1$ (with weight $y^{2} D_{i, i+1}+E_{i, i+1}$ ), $i \rightarrow i$ (with weight $y^{2} D_{i, i}+E_{i, i}$ ), or $i \rightarrow i-1$ (with weight $y^{2} D_{i, i-1}+E_{i, i-1}$ ).

Note that these kind of walks in $\mathbb{N}$ are clearly in bijection with Motzkin paths (see the next section for more on the combinatorics of these paths). By the standard methods of Flajolet [12], this gives a continued fraction for the generating function. We use the notation $\frac{a_{1}}{b_{1}}-\frac{a_{2}}{b_{2}}-\cdots=\left(a_{1} /\left(b_{1}-\right.\right.$ $\left.\left.\left(a_{2} /\left(b_{2}-\cdots\right)\right)\right)\right)$ for continued fractions.

Theorem 6.1. Let $\gamma_{h}=y^{2}[h+1]_{q}+[h]_{q}+y t q^{h}\left([h]_{q}+[h+1]_{q}\right)$ for $h \geq 0$ and $\lambda_{h}=y[h]_{q}^{2}\left(y+t q^{h-1}\right)\left(1+y t q^{h}\right)$ for $h \geq 1$, then we have:

$$
\sum_{n \geq 0} B_{n}(y, t, q) z^{n}=\frac{1}{1-\gamma_{0} z}-\frac{\lambda_{1} z^{2}}{1-\gamma_{1} z}-\frac{\lambda_{2} z^{2}}{1-\gamma_{2} z}-\cdots
$$


Crossings of signed permutations and $q$-Eulerian numbers of type $B 209$

Proof. This essentially follows the above discussion about the transfer matrix method and Motzkin paths. It suffices to check $\gamma_{h}=y^{2} D_{h, h}+E_{h, h}$ and $\lambda_{h}=\left(y^{2} D_{h-1, h}+E_{h-1, h}\right) E_{h, h-1}$.

In particular, we have

$$
\begin{aligned}
\sum_{n \geq 0} B_{n}(y, 1,-1) z^{n} & =\frac{1}{1-\left(y+y^{2}\right) z-\frac{1-y(y+1)(1-y) z^{2}}{1-(1-y) z}}=\frac{1-z+z y}{1-z-y^{2} z} \\
& =1+\frac{\left(y+y^{2}\right) z}{1-\left(1+y^{2}\right) z} .
\end{aligned}
$$

It is then easy to obtain

$$
B_{n}(y, 1,-1)=\sum_{k=1}^{2 n} y^{k}\left(\begin{array}{c}
n-1 \\
\lceil k / 2\rceil-1
\end{array}\right) .
$$

Using (6), we thus obtain as mentioned in Theorem 2.1 that the value at $q=-1$ of the $q$-Eulerian numbers of type $B$ are binomial coefficients:

$$
\begin{aligned}
E_{n, k}^{B}(-1) & =\left[y^{2 k}\right] B_{n}(y, 1,-1)+\left[y^{2 k+1}\right] B_{n}(y, 1,-1) \\
& =\left(\begin{array}{l}
n-1 \\
k-1
\end{array}\right)+\left(\begin{array}{c}
n-1 \\
k
\end{array}\right)=\left(\begin{array}{l}
n \\
k
\end{array}\right) .
\end{aligned}
$$

\subsection{Solution 2}

Let $X=\left(X_{i, j}\right)_{i, j \geq 0}$ and $Y=\left(Y_{i, j}\right)_{i, j \geq 0}$ where

$X_{i, i+1}=[i+1]_{q}$ and $X_{i, j}=0$ otherwise, $\quad Y_{i+1, i}=1$ and $Y_{i, j}=0$ otherwise,

and

$$
\left\langle W\left|=\left(1, y t,(y t)^{2}, \ldots\right), \quad\right| V\right\rangle=(1,0,0, \ldots)^{\mathrm{t}} .
$$

We can check that $X Y-q Y X=I$ and that $D=X(I+Y)$ and $E=Y X(I+$ $Y)$ together with $\langle W|$ and $|V\rangle$ provide a solution of (7). The coefficients are:

$$
\begin{aligned}
& D_{i, i}=D_{i, i+1}=[i+1]_{q}, \quad D_{i, j}=0 \text { otherwise, } \\
& E_{i, i-1}=E_{i, i}=[i]_{q}, \quad E_{i, j}=0 \text { otherwise. }
\end{aligned}
$$

Theorem 6.2. We have $B_{0}=1$, and the recurrence relation:

$$
B_{n}(y, t, q)=(y+t) D_{q}\left[(1+y t) B_{n-1}(y, t, q)\right],
$$

where $D_{q}$ is the $q$-derivative with respect to $t$, which sends $t^{n}$ to $[n]_{q} t^{n-1}$. 
Proof. Each polynomial $P$ in $y, t$, and $q$ can be uniquely written $\sum_{k \geq 0} p_{k}(y t)^{k}$ where the coefficients $p_{k}$ are functions of $y$ and $q$ (possibly rational function). We can identify the polynomial $P$ with the vector of coefficients $v=\left(p_{1}, p_{2}, \ldots\right)$. More precisely, the correspondence is $P=\langle W| \cdot v$. Under this identification $P \leftrightarrow v$, we can see that multiplication by $y t$ on polynomials corresponds to the matrix $Y$ acting on vectors, and $y^{-1} D_{q}$ acting on polynomials corresponds to the matrix $X$. This means:

$$
(\langle W| Y) \cdot v=y t(\langle W| \cdot v), \quad\langle W| \cdot(X v)=y^{-1} D_{q}[\langle W| \cdot v] .
$$

From the definition of $D$ and $E$ in terms of $X$ and $Y$, we have $y^{2} D+E=$ $\left(y^{2} I+Y\right) X(I+Y)$. Then:

$$
\begin{aligned}
B_{n}(y, t, q) & =\left\langle W\left|\left(y^{2} D+E\right)^{n}\right| V\right\rangle=\left\langle W\left|\left(\left(y^{2} I+Y\right) X(I+Y)\right)^{n}\right| V\right\rangle \\
& =(y+t) D_{q}\left[(1+y t)\left\langle W\left|\left(y^{2} D+E\right)^{n-1}\right| V\right\rangle\right]
\end{aligned}
$$

and the result follows.

We can also see $y^{2} D+E$ as a transfer matrix in this case. This leads to the following definition.

Definition 1. A Motzkin suffix of length $n$ and starting height $k$ is a path in $\mathbb{N}^{2}$ from $(0, k)$ to $(n, 0)$ with steps $(1,1),(1,0)$ and $(1,-1)$, respectively denoted $\nearrow, \rightarrow$ and $\searrow$. The Motzkin paths are the particular cases where the starting height is 0 . We denote by $\operatorname{sh}(p)$ the starting height of the path $p$.

Proposition 6.3. $B_{n}(y, t, q)$ is the weighted generating functions of Motzkin suffixes of length $n$, where the weight of a path $p$ is the product of $(y t)^{\operatorname{sh}(p)}$, and:

- $y^{2}[h+1]_{q}$ for each step $\nearrow$ from height $h$ to $h+1$,

- $y^{2}[h+1]_{q}+[h]_{q}$ for each step $\rightarrow$ at height $h$,

- $[h+1]_{q}$ for each step $\searrow$ from height $h+1$ to $h$.

Proof. Let us write $B_{n}(y, t, q)=\sum_{k \geq 0} p_{k}(y t)^{k}$ where the coefficients $p_{k}$ are functions of $y$ and $q$. From $B_{n}(y, t, q)=\left\langle W\left|\left(y^{2} D+E\right)^{n}\right| V\right\rangle$, we see that $p_{k}$ is the coefficient $(k, 0)$ of the matrix $\left(y^{2} D+E\right)^{n}$. The transfer matrix method shows that $p_{k}$ is the weighted generating function of walks in $\mathbb{N}$ from $k$ to 0 , with the following steps: either $i \rightarrow i+1$ (with weight $y^{2} D_{i, i+1}+E_{i, i+1}$ ), $i \rightarrow i$ (with weight $y^{2} D_{i, i}+E_{i, i}$ ), or $i \rightarrow i-1$ (with weight $y^{2} D_{i, i-1}+E_{i, i-1}$ ). These walks in $\mathbb{N}$ are clearly in bijection with Motzkin suffixes, so the result follows. 
Crossings of signed permutations and $q$-Eulerian numbers of type $B 211$

See the next section for more on the combinatorics of these weighted Motzkin suffixes.

\section{Interpretation of $B_{n}(y, t, q)$ via weighted Motzkin paths}

We show here that some known bijections from [6] can be adapted to the case of signed permutations. In this reference, the first author obtains refinements of two bijections originally given by Françon and Viennot [15], Foata and Zeilberger [14]. In the case of signed permutations, we will see that each of these two bijections has two variants, corresponding to the two kinds of paths obtained in the previous section.

The two variants of the Françon-Viennot bijection give new interpretations $B_{n}(y, t, q)$ in terms ascent-like and pattern-like statistics in signed permutations. On the other side, the two variants of the Foata-Zeilberger bijection permit to recover the known interpretation as in (5) (so we omit details in this case).

\subsection{Weighted Motzkin paths}

Let $\mathcal{M}_{n}$ be the set of weighted Motzkin paths of length $n$, where each step is either:

- $\nearrow$ from height $h$ to $h+1$ with weight $q^{i}, 0 \leq i \leq h$ (type 1 ),

- $\nearrow$ from height $h$ to $h+1$ with weight $y t q^{h+1+i}, 0 \leq i \leq h$ (type 2),

- $\rightarrow$ at height $h$ with weight $y^{2} q^{i}, 0 \leq i \leq h$ (type 3 ),

- $\rightarrow$ at height $h$ with weight $y t q^{h+i}, 0 \leq i \leq h$ (type 4 ),

- $\rightarrow$ at height $h$ with weight $q^{i}, 0 \leq i \leq h-1$ (type 5),

- $\rightarrow$ at height $h$ with weight $y t q^{h+i}, 0 \leq i \leq h-1$ (type 6),

- \from height $h+1$ to $h$ with weight $y^{2} q^{i}, 0 \leq i \leq h$ (type 7),

- \from height $h+1$ to $h$ with weight $y t q^{h+i}, 0 \leq i \leq h$ (type 8).

This set has generating function $B_{n}(y, t, q)$ since the weights correspond to coefficients of $y^{2} D+E$, where we use the first solution of the Matrix Ansatz from the previous section. More precisely, the correspondence is: $E_{h, h-1} \rightarrow$ types 1 and $2, D_{h, h} \rightarrow$ type $3, E_{h, h} \rightarrow$ types 4,5 and $6, D_{h, h+1} \rightarrow$ type 7 , $E_{h, h+1} \rightarrow$ type 8 . Note that the weight on a step does not always determine its type (compare type 4 and 6 ), so that we need to think in terms of weighted paths where each step has a label between 1 and 8 to indicate its type. 

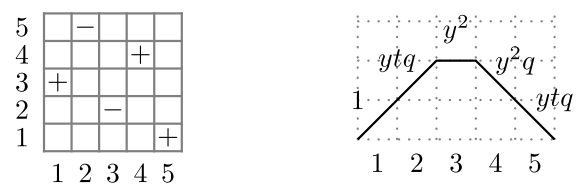

Figure 15: The first variant of the Françon-Viennot bijection, with the signed permutation $\pi=3,-5,-2,4,1$.

7.1.1. The Françon-Viennot bijection, first variant There is a bijection between $S_{n}$ and weighted Motzkin paths which follows the number of descents and the number of 31-2 patterns in a permutation [6]. Let $\sigma \in S_{n}$, then its image path is obtained by "scanning" the graph of $\sigma$ from bottom to top, i.e. the $i$ th step is obtained by examining $\sigma^{-1}(i)$. In the case of a signed permutation $\pi$, we use the representation as in Figure 15: we place a + or a - in the square in the $i$ th column and $\left|\pi_{i}\right|$ th row depending on the sign of $\pi(i)$.

Definition 2. For any $\sigma \in S_{n}$ and $1 \leq i \leq n$, let 31-2( $\left.\sigma, i\right)=\#\{j: 1 \leq j<$ $i-1$ and $\left.\pi_{j}>\pi_{i}>\pi_{j+1}\right\}$, and let 2-31 $(\sigma, i)=\#\left\{j: i<j<n\right.$ and $\pi_{j}>$ $\left.\pi_{i}>\pi_{j+1}\right\}$. Let also $31-2(\sigma)=\sum_{i=1}^{n} 31-2(\sigma, i)$.

Let $\pi \in B_{n}$. We denote by $|\pi|$ the permutation obtained by removing the negative signs of $\pi$, that is, $|\pi|_{i}=\left|\pi_{i}\right|$ for $i=1,2, \ldots, n$. We take the convention that $\pi_{0}=0$ and $\pi_{n+1}=n+1$. The bijection is defined in the following way. The path corresponding to $\pi$ is of length $n$ such that, if $j=\left|\pi_{i}\right|$ and denoting $s$ the $j$ th step, we have:

- If $\left|\pi_{i-1}\right|>\left|\pi_{i}\right|<\left|\pi_{i+1}\right|$ and $\pi_{i}>0$, then $s$ is of type 1 with weight $q^{31-2(|\pi|, i)}$.

- If $\left|\pi_{i-1}\right|>\left|\pi_{i}\right|<\left|\pi_{i+1}\right|$ and $\pi_{i}<0$, then $s$ is of type 2 with weight $y t q^{h+1+31-2(|\pi|, i)}$.

- If $\left|\pi_{i-1}\right|<\left|\pi_{i}\right|<\left|\pi_{i+1}\right|$ and $\pi_{i}>0$, then $s$ is of type 3 with weight $y^{2} q^{31-2(|\pi|, i)}$.

- If $\left|\pi_{i-1}\right|<\left|\pi_{i}\right|<\left|\pi_{i+1}\right|$ and $\pi_{i}<0$, then $s$ is of type 4 with weight $y t q^{h+31-2(|\pi|, i)}$.

- If $\left|\pi_{i-1}\right|>\left|\pi_{i}\right|>\left|\pi_{i+1}\right|$ and $\pi_{i}>0$, then $s$ is of type 5 with weight $q^{31-2(|\pi|, i)}$.

- If $\left|\pi_{i-1}\right|>\left|\pi_{i}\right|>\left|\pi_{i+1}\right|$ and $\pi_{i}<0$, then $s$ is of type 6 with weight $y t q^{h+31-2(|\pi|, i)}$.

- If $\left|\pi_{i-1}\right|<\left|\pi_{i}\right|>\left|\pi_{i+1}\right|$ and $\pi_{i}>0$, then $s$ is of type 7 with weight $y^{2} q^{31-2(|\pi|, i)}$. 
Crossings of signed permutations and $q$-Eulerian numbers of type $B 213$

- If $\left|\pi_{i-1}\right|<\left|\pi_{i}\right|>\left|\pi_{i+1}\right|$ and $\pi_{i}<0$, then $s$ is of type 8 with weight $y t q^{h+31-2(|\pi|, i)}$.

In the case where $\pi$ has no negative entry, this defines a bijection with the paths having steps of types 1, 3, 5, 7 only. This is a result from [6], and what we present here is a variant, so we refer to this work for more details. In the case of signed permutations, since each entry can be negated, it is natural that each step of type $(1,3,5$, or 7$)$ has a respective variant (type $2,4,6$, or 8$)$. So the fact that the map is a bijection can be deduced from the case of unsigned permutations. It is also clear that $t$ follows the number of negative entries of $\pi$ since there is a factor $t$ only in steps of type $2,4,6$, and 8 .

Let us check what is the statistic followed by $y$. There is a factor $y$ on each step of type $2,4,6,8$ so that this statistic is the sum of neg $(\pi)$ and other terms coming from the factors $y^{2}$ in steps of type 3 and 7 . Note that the $j$ th step is of type 3 or 7 if and only if $\left|\pi_{i-1}\right|<\pi_{i}$. This leads us to define an ascent $\operatorname{statistic} \operatorname{hasc}(\pi)$ as

$$
\operatorname{hasc}(\pi)=2 \times \#\left\{i: 0 \leq i \leq n-1 \text { and }\left|\pi_{i}\right|<\pi_{i+1}\right\}+\operatorname{neg}(\pi) .
$$

It remains only to check what is the statistics followed by $q$. Since there is

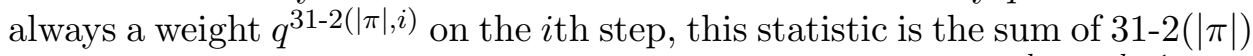
and other terms. It remains to take into account the weights $q^{h}$ or $q^{h+1}$ that appear on each step of type 2, 4, 6, 8. From the properties of the bijection in the unsigned case, we have that the "minimal" height $h$ of the $i$ th step is $31-2(|\pi|, i)+2-31(|\pi|, i)$, plus 1 in the case where $\left|\pi_{i-1}\right|>\left|\pi_{i}\right|>\left|\pi_{i+1}\right|$. So, apart a factor $q$ on each step of type 2 and 6 , we obtain the statistic

$$
31-2(|\pi|)+\sum_{\substack{1 \leq i \leq n \\ \pi_{i}<0}}(31-2(|\pi|, i)+2-31(|\pi|, i)) .
$$

Note that to take into account the factor $q$ on each step of type 2 and 6 , we can count $i$ such that $1 \leq i<n$ and $\left|\pi_{i-1}\right|>-\pi_{i}>0$. The statistic we eventually obtain can be rearranged as follows:

$$
\begin{gathered}
\operatorname{pat}(\pi)=\#\left\{(i, j): 1 \leq i<j \leq n, \text { and }\left|\pi_{i}\right|>\left|\pi_{j}\right|>\left|\pi_{i+1}\right|\right\}+ \\
\\
\#\left\{(i, j): 1 \leq i, j \leq n, \text { and }\left|\pi_{i}\right|>-\pi_{j} \geq\left|\pi_{i+1}\right|\right\} .
\end{gathered}
$$

This is a "pattern" statistic that is somewhat similar to the 31-2 statistic of the unsigned case (and indeed, identical if $\pi$ has only positive entries). Eventually, our first variant of the Françon-Viennot bijection gives the following. 

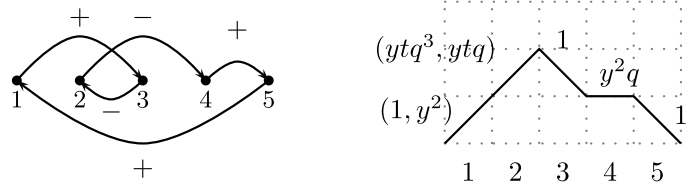

Figure 16: The first variant of the Foata-Zeilberger bijection, with $\pi=$ $3,-4,-2,5,1$.

Proposition 7.1. With hasc $(\pi)$ defined as in (19), we have:

$$
B_{n}(y, t, q)=\sum_{\pi \in B_{n}} y^{\operatorname{hasc}(\pi)} t^{\operatorname{neg}(\pi)} q^{\operatorname{pat}(\pi)} .
$$

Note that in the case $t=0$, we recover the known result [28]:

$$
\sum_{k=1}^{n} y^{k} E_{n, k}(q)=\sum_{\sigma \in S_{n}} y^{\operatorname{asc}(\sigma)+1} q^{31-2(\sigma)}
$$

where $\operatorname{asc}(\sigma)=n-1-\operatorname{des}(\sigma)=\#\left\{i: 1 \leq i \leq n-1\right.$ and $\left.\sigma_{i}<\sigma_{i+1}\right\}$.

7.1.2. The Foata-Zeilberger bijection, first variant In case of unsigned permutations, this bijection follows the number of weak excedances and crossings, see the bijection $\Psi_{F Z}$ in [6]. To extend it, we use a representation of a signed permutation as an arrow diagram (this is equivalent to the pignose diagrams used earlier: if $\sigma \in S_{n}$, we put $n$ dots in a row and draw an arrow from $i$ to $\sigma(i)$ which is above the axis if $i \leq \sigma(i)$ and below otherwise, see [6]). The idea is to draw the arrow diagram of $|\pi|$ and label an arrow from $i$ to $|\pi(i)|$ by + or - depending on the sign of $\pi(i)$, see Figure 16 .

The crossings can be read in this representation as follows. The proof can be done by distinguishing all the possible cases for the signs of $i$ and $j$ for each type of crossing $(i, j)$. We omit details.

Proposition 7.2. Each crossing $(i, j)$ in the signed permutation $\pi$ corresponds to one of the six configurations in Figure 17, where \pm means that the label of the arrow can be either + or - , and the dots indicate that there might be an equality of the endpoint of an arrow and the startpoint of the other arrow.

For example, let us consider the signed permutation in Figure 16. The dots $1,2,3$ (respectively, 1, 2, 5 and 1, 2, 3, 5) correspond to a crossing as 

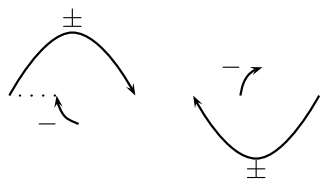
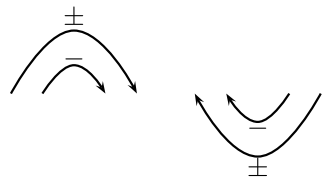
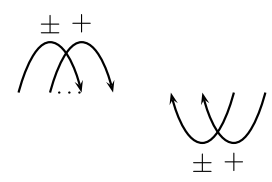

Figure 17: The list of configurations characterizing a crossing in the arrow diagram of a signed permutation.

in the first (respectively, second and fourth) configuration of Figure 17. The dots 2, 4 are also a crossing as in (the limit case of) the first configuration, and eventually the dots $2,4,5$ are a crossing as in (the limit case of) the fifth configuration. So the signed permutation $3,-4,-2,5,1$ has 5 crossings.

Once we have this new description of the crossings, it is possible to encode the arrow diagram as in Figure 16 by elements in $\mathcal{M}_{n}$. Actually it will be more convenient to see these paths in a slightly different way: each step $\nearrow$ (with weight $a$ ) faces a step $\searrow$ (weight $b$ ), but now we think as if the step $\searrow$ have weight 1 and the facing step $\nearrow$ has weight $(a, b)$. But we still consider the steps $\rightarrow$ of types $3,4,5,6$ as before.

Now, the path is obtained from the signed permutation by "scanning" the arrow diagram from right to left, so that after scanning $i$ nodes in the diagram, we have built a Motzkin suffix of length $i$. Suppose that there are $h$ unconnected strands after scanning these $i$ nodes, and accordingly, the Motzkin suffix starts at height $h$. When scanning the following node, we have several possibilities:

- If the $i$ th node is $>$, then we add a step $\searrow$ (with weight 1 ) to the Motzkin suffix,

- If the $i$ th node is $\frac{}{8}$ or $\downarrow$, then we add a step $\rightarrow$ (type 3 ) with weight $y^{2} q^{i}$ where $i$ counts the number of crossings as in the 5 th configuration that appear when adding this $i$ th node to the ones at its right.

- If the $i$ th node is $\overline{\boldsymbol{8}}$, then we add a step $\rightarrow$ (type 4 ) with weight $y t$ to the Motzkin suffix, and if it is $\overline{\boldsymbol{\gamma}}$, then we add a step $\rightarrow$ (type 4 ) with weight $y t q^{h+i}$, where $i$ counts the number of crossings as in 3rd configuration that appear when adding this $i$ th node to the ones to its right. Note that $h$ is the number of crossings as in 2 nd configuration that appear.

- If the $i$ th node is $\mathbf{\lambda}$, then we add a step step $\rightarrow$ (type 5) with weight $q^{i}$ where $i$ counts the number of crossings as in the 6 th configuration that appear. 
- If the $i$ th node is $\boldsymbol{\gamma}$, then we add a step $\rightarrow$ (type 6$)$ with weight $y t q^{h+i}$ where $i$ counts the number of crossings as in the 4th configuration that appear. Note that $h$ is the number of crossings as in 1st configuration that appear.

- If the $i$ th node is $\measuredangle$, then we add a step $\nearrow$ with a weight $(a, b)$ where $a$ and $b$ are as follows (this is similar to the previous cases, but here we need to encode information about both the ingoing and outgoing arrow). The possibilities for $a$ are $q^{0}, \ldots, q^{h-1}$, or $y t q^{h}, \ldots, y t q^{2 h-1}$, such that there is a factor 1 (resp. $y t$ ) if the label of the ingoing arrow is + (resp. - ), and $q$ counts the crossings of the 6th (resp. 1st and 4th) configuration. The possibilities for $b$ are $y^{2} q^{0}, \ldots, y^{2} q^{h-1}$, or $y t q^{h-1}, \ldots, y t q^{2 h-2}$, such that there is a factor $y^{2}$ (resp. $y t$ ) if the label of the outgoing arrow is + (resp. - ), and $q$ counts the crossings of the 5th (resp. 2nd and 3rd) configuration.

See Figure 16 for an example. Let $i<j$, if there is an arrow from $i$ to $j$ with a label + , then the $i$ th step gets a weight $y^{2}$, and if there is an arrow from $i$ to $j$ or from $j$ to $i$ with a label - , then the $i$ th step gets a weight $y t$. It follows that the parameters $y$ and $t$ correspond to fwex $(\pi)$ and $\operatorname{neg}(\pi)$ in signed permutations. By the design of the bijection, the parameter $q$ corresponds to $\operatorname{cr}(\pi)$. Hence we recover (5).

\subsection{Suffixes of weighted Motzkin paths}

We can see a signed permutation as a permutation on $[ \pm n]$ or on $[2 n]$ with a symmetry property. Then, applying the bijections of the unsigned case gives some weighted Motzkin paths with a vertical symmetry. It is natural to keep only the second half a vertically-symmetric path and obtain suffixes of Motzkin paths.

Definition 3. Let $\mathcal{N}_{n}$ be the set of weighted Motzkin suffixes with weights:

- $y^{2} q^{i}$ with $0 \leq i \leq h$, for a step $\nearrow$ at height $h$ to $h+1$,

- either $y^{2} q^{i}$ with $0 \leq i \leq h$, or $q^{i}$ with $0 \leq i \leq h-1$, for a step $\rightarrow$ at height $h$,

- $q^{i}$ with $0 \leq i \leq h$, for a step $\searrow$ at height $h+1$ to $h$.

For any $p \in \mathcal{N}_{n}$, let $\operatorname{sh}(p)$ be its initial height, and let $\mathrm{w}(p)$ be its total weight, i.e. the product of the weight of each step.

Then $B_{n}(y, t, q)$ is the generating function $\sum_{p \in \mathcal{N}_{n}}(y t)^{\operatorname{sh}(p)} \mathrm{w}(p)$. This is a consequence of Proposition 6.3; the only difference is that instead of a step $\nearrow$ with weight $y^{2}[h+1]_{q}$, we consider $h+1$ different kind of steps with weight $y^{2} q^{0}, \ldots, y^{2} q^{h}$ (and similarly for the other steps). 
Crossings of signed permutations and $q$-Eulerian numbers of type $B 217$
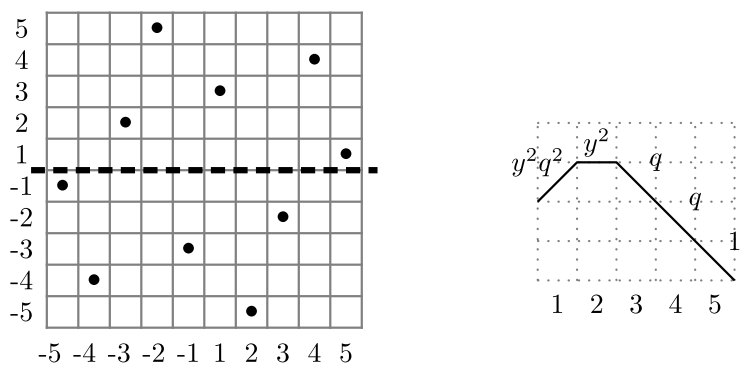

Figure 18: The second variant of the Françon-Viennot bijection, with $\pi=$ $3,-5,-2,4,1$.

7.2.1. The Françon-Viennot bijection, second variant This second variant is a bijection between $\mathcal{N}_{n}$ and $B_{n}$, and it gives a combinatorial model of $B_{n}(y, t, q)$ involving the flag descents and different from the previous ones. The second bijection is done using the diagram of a signed permutation $\pi \in B_{n}$ as in Figure 18. More precisely, we have a $2 n \times 2 n$ array, lines and columns are numbered with integers in $[ \pm n]$ in increasing order, and there is a dot at each cell of coordinates $(i, \pi(i))$ for $i \in[ \pm n]$.

Definition 4. For a signed permutation $\pi$, let fneg $(\pi)$ be the number of positive integers followed by a negative integer in the sequence $\pi(-n), \ldots, \pi(-1)$, $\pi(1), \ldots, \pi(n)$. Note that fneg $(\pi)=0$ if and only if $\pi$ is actually an unsigned permutation. Let

$$
31-2^{+}(\pi)=\sum_{\substack{1 \leq i \leq 2 n \\ \tilde{\pi}(i)>n}} 31-2(\tilde{\pi}, i)
$$

where $\tilde{\pi} \in S_{2 n}$ is the permutation corresponding to $\pi$ via the order-preserving identification $[ \pm n] \rightarrow[2 n]$.

The bijection is defined as follows. We take here the convention that $\pi_{-n-1}=-n-1$ and $\pi_{n+1}=n+1$. Let $j \in[n]$, let $i \in[ \pm n]$ such that $\pi_{i}=j$, then:

- if $\pi_{i-1}<\pi_{i}<\pi_{i+1}$ or $\pi_{i-1}>\pi_{i}>\pi_{i+1}$, the $j$ th step is $\rightarrow(i=2$ in the example),

- if $\pi_{i-1}>\pi_{i}<\pi_{i+1}$, the $j$ th step is $\nearrow(i=1$ in the example),

- if $\pi_{i-1}<\pi_{i}>\pi_{i+1}$, the $j$ th step is $\searrow(i=3,4,5$ in the example).

And the weight of the $i$ th step is $y^{2} q^{31-2(\tilde{\pi}, i+n)}$ if $\pi_{i}>\pi_{i+1}, q^{31-2(\tilde{\pi}, i+n)}$ otherwise. 

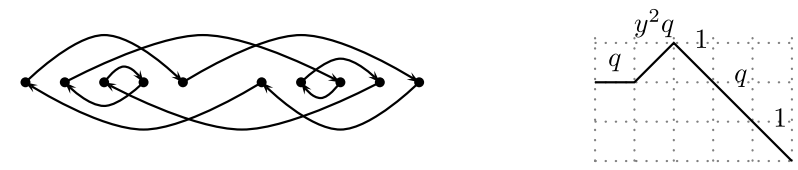

Figure 19: The second variant of the Foata-Zeilberger bijection in the case of $\pi=-5,4,2,-3,1$.

The properties of the Françon-Viennot bijection from [6] show that, denoting $p$ the image of $\pi$ under this bijection, we have:

$$
(y t)^{\operatorname{sh}(p)} \mathrm{w}(p)=y^{2 n-\mathrm{fdes}(\pi)} t^{\mathrm{fneg}(\pi)} q^{31-2^{+}(\pi)} .
$$

Let us denote $\operatorname{fasc}(\pi)=2 n-1-\operatorname{fdes}(\pi)$, which can be interpreted as an ascent statistic. Indeed, in the sequence $-\pi_{n}, \ldots,-\pi_{1}, \pi_{1}, \ldots, \pi_{n}, \operatorname{fdes}(\pi)$ (resp. fasc $(\pi)$ ) is the number of integers followed by a smaller (resp. greater) integer. So the bijection proves:

Proposition 7.3. For $n \geq 1$,

$$
B_{n}(y, t, q)=\sum_{\pi \in B_{n}} y^{\mathrm{fasc}(\pi)+1} t^{\mathrm{fneg}(\pi)} q^{31-2^{+}(\pi)} .
$$

Note that once again, we recover $(21)$ in the case $t=0$.

7.2.2. The Foata-Zeilberger bijection, second variant Let $\pi \in B_{n}$, we use here the arrow diagram as in Figure 19 where the dots are labeled by $-n, \ldots,-1,1, \ldots, n$ and there is an arrow from $i$ to $\pi(i)$ above the axis if $i \leq \pi(i)$ and below otherwise (this is equivalent to the full pignose diagram used in Section 5 where each pignose collapses to a single dot). The path $p \in \mathcal{N}_{n}$ is as follows. If at the $i$ th node in the diagram (where $1 \leq i \leq n$ ), there is an arrow arriving from the left and an arrow going to the left, then the $i$ th step is $\searrow$. If there is an arrow going to the right and arriving from the right, then it is a step $\nearrow$. In all other cases, it is a step $\rightarrow$. Then, for each arrow going from $i$ to $j$ with $0<i<j$, we give a weight $y^{2}$ to the $i$ th step in the path; for each crossing $i<j \leq \pi(i)<\pi(j)$ with $j>0$, we give a weight $q$ to the $j$ th step; and for each crossing $i>j>\pi(i)>\pi(j)$ with $j>0$, we give a weight $q$ to the $j$ th step. See Figure 19 for example.

The number of arrows that join a positive integer to a negative one is $\operatorname{neg}(\pi)$. From the construction, this is also the initial height of the path we have built. So giving a weight $(y t)^{\operatorname{sh}(p)}$ to the path $p$ ensures that $y$ and 
$t$ respectively follow fwex $(\pi)$ and neg $(\pi)$. Also from the definition of the bijection, $q$ counts the number of crossings. Hence we recover (5). We do not give more details since this bijection does not give new statistics on signed permutations.

\section{Formula for $B_{n}(y, 1, q)$}

In this section, we prove a formula for $B_{n}(y, 1, q)$. The formula itself is somewhat similar to the one for $B_{n}(y, 0, q)$ in Equation (3). The result for $B_{n}(y, 0, q)$ was proved in [17], but the present proof for $B_{n}(y, 1, q)$ is different.

\section{Theorem 8.1.}

$$
\begin{aligned}
B_{n}(y, 1, q)= & \frac{1}{(1-q)^{n}} \sum_{j=0}^{n}(-1)^{j}\left(\sum_{i=0}^{2 n-2 j} y^{i}\left(\begin{array}{c}
n \\
j+\left\lceil\frac{i}{2}\right\rceil
\end{array}\right)\left(\begin{array}{c}
n \\
\left\lfloor\frac{i}{2}\right\rfloor
\end{array}\right)\right) \\
& \times\left(\sum_{\ell=0}^{2 j} y^{\ell} q^{\frac{\ell(2 j-\ell+1)}{2}}\right) .
\end{aligned}
$$

Or, equivalently:

$$
\begin{aligned}
B_{n}(y, 1, q)= & \frac{1}{(1-q)^{n}} \sum_{k=0}^{n}\left(\sum_{i=0}^{n-k} y^{2 i}\left(\left(\begin{array}{c}
n \\
i
\end{array}\right)\left(\begin{array}{c}
n \\
i+k
\end{array}\right)-\left(\begin{array}{c}
n \\
i-1
\end{array}\right)\left(\begin{array}{c}
n \\
i+k+1
\end{array}\right)\right)\right) \\
& \times \sum_{j=0}^{k} y^{k-j}(-1)^{j} \sum_{\ell=0}^{2 j} y^{\ell} q^{\frac{\ell(2 j-\ell+1)}{2}} .
\end{aligned}
$$

We can obtain (23) from (24) by simplifying a summation as follows. Let $P_{j}=\sum_{\ell=0}^{2 j} y^{\ell} q^{\frac{\ell(2 j-\ell+1)}{2}}$, then the right-hand side of (24) is

$$
\begin{aligned}
& \frac{1}{(1-q)^{n}} \sum_{j=0}^{n}(-1)^{j} P_{j} \sum_{k=j}^{n}\left(\sum_{i=0}^{n-k} y^{k-j+2 i}\left(\left(\begin{array}{c}
n \\
i
\end{array}\right)\left(\begin{array}{c}
n \\
i+k
\end{array}\right)-\left(\begin{array}{c}
n \\
i-1
\end{array}\right)\left(\begin{array}{c}
n \\
i+k+1
\end{array}\right)\right)\right) \\
& \left.=\frac{1}{(1-q)^{n}} \sum_{j=0}^{n}(-1)^{j} P_{j} \sum_{m=0}^{2 n-2 j} y^{m} \sum_{i=0}^{\lfloor m / 2\rfloor}\left(\begin{array}{c}
n \\
i
\end{array}\right)\left(\begin{array}{c}
n \\
m+j-i
\end{array}\right)-\left(\begin{array}{c}
n \\
i-1
\end{array}\right)\left(\begin{array}{c}
n \\
m+j-i+1
\end{array}\right)\right) .
\end{aligned}
$$

Here we have introduced the new index $m=2 i+k-j$, and the condition $k-j \geq 0$ gives the condition $m \geq 2 i$, i.e. $i \leq\lfloor m / 2\rfloor$. But the $i$-sum in the latter formula is actually telescopic and only the term $\left(\begin{array}{l}n \\ i\end{array}\right)\left(\begin{array}{c}n \\ m+j-i\end{array}\right)$ with 
$i=\lfloor m / 2\rfloor$ remains. Using the fact that $m-\lfloor m / 2\rfloor=\lceil m / 2\rceil$, we obtain (23).

So, (23) is a simpler formula, but (24) is the one which is conveniently proved, using results from [18]. The theorem follows from the two lemmas below (and a third lemma is needed to prove the second lemma). The first lemma was essentially present in [19].

Lemma 8.2. Suppose that two sequences $\left(b_{n}\right)_{n \geq 0}$ and $\left(c_{n}\right)_{n \geq 0}$ are such that:

$$
\sum_{n \geq 0} b_{n} z^{n}=\frac{1}{1-\gamma_{0} z}-\frac{\lambda_{1} z^{2}}{1-\gamma_{1} z}-\frac{\lambda_{2} z^{2}}{1-\gamma_{2} z}-\cdots
$$

and

$$
\begin{aligned}
\sum_{n \geq 0} c_{n} z^{n}= & \frac{1}{(1+z)\left(1+y^{2} z\right)-\gamma_{0} z}-\frac{\lambda_{1} z^{2}}{(1+z)\left(1+y^{2} z\right)-\gamma_{1} z}- \\
& \frac{\lambda_{2} z^{2}}{(1+z)\left(1+y^{2} z\right)-\gamma_{2} z}-\cdots
\end{aligned}
$$

Then we have:

$$
b_{n}=\sum_{k=0}^{n}\left(\sum_{j=0}^{n-k} y^{2 j}\left(\left(\begin{array}{c}
n \\
j
\end{array}\right)\left(\begin{array}{c}
n \\
j+k
\end{array}\right)-\left(\begin{array}{c}
n \\
j-1
\end{array}\right)\left(\begin{array}{c}
n \\
j+k+1
\end{array}\right)\right)\right) c_{k} .
$$

Proof. Let $f(z)$ and $g(z)$ respectively denote the generating functions of $\left(b_{n}\right)_{n \geq 0}$ and $\left(c_{n}\right)_{n \geq 0}$ as in Equations (25) and (26). Divide by $(1+z)(1+$ $\left.y^{2} z\right)$ the numerator and denominator of each fraction in (26), this gives an equivalence of continued fractions so that:

$$
z g(z)=\frac{z}{(1+z)\left(1+y^{2} z\right)} f\left(\frac{z}{(1+z)\left(1+y^{2} z\right)}\right)
$$

It follows that

$$
z f(z)=C(z) g(C(z))
$$

where $C(z)$ is the compositional inverse of $\frac{z}{(1+z)\left(1+y^{2} z\right)}$. It remains to show that

$$
C(z)^{k+1}=\sum_{n \geq 0}\left(\sum_{j=0}^{n-k} y^{2 j}\left(\left(\begin{array}{c}
n \\
j
\end{array}\right)\left(\begin{array}{c}
n \\
j+k
\end{array}\right)-\left(\begin{array}{c}
n \\
j-1
\end{array}\right)\left(\begin{array}{c}
n \\
j+k+1
\end{array}\right)\right)\right) z^{n+1}
$$


Crossings of signed permutations and $q$-Eulerian numbers of type $B 221$

Indeed, the lemma follows from taking the coefficient of $z^{n+1}$ in both sides of (27) and using (28) to simplify the right-hand side. Showing (28) can be conveniently done using Lagrange inversion. For example, with [5, p.148, Theorem A], we obtain

$$
\begin{aligned}
{\left[z^{n+1}\right] C(z)^{k+1} } & =\frac{k+1}{n+1}\left[z^{n-k}\right]\left((1+z)\left(1+y^{2} z\right)\right)^{n+1} \\
& =\frac{k+1}{n+1} \sum_{j=0}^{n-k} y^{2 j}\left(\begin{array}{c}
n+1 \\
j
\end{array}\right)\left(\begin{array}{c}
n+1 \\
n-k-j
\end{array}\right),
\end{aligned}
$$

and it is straightforward to check that

$$
\frac{k+1}{n+1}\left(\begin{array}{c}
n+1 \\
j
\end{array}\right)\left(\begin{array}{c}
n+1 \\
n-k-j
\end{array}\right)=\left(\begin{array}{c}
n \\
j
\end{array}\right)\left(\begin{array}{c}
n \\
j+k
\end{array}\right)-\left(\begin{array}{c}
n \\
j-1
\end{array}\right)\left(\begin{array}{c}
n \\
j+k+1
\end{array}\right) .
$$

This completes the proof.

We can apply this lemma with $b_{n}=(1-q)^{n} B_{n}(y, 1, q)$. The continued fraction expansion of $\sum b_{n} z^{n}$ is immediately obtained from the one of $\sum B_{n}(y, t, q) z^{n}$ obtained in Theorem 6.1. More precisely, it is exactly Equation (25) with the values

$$
\begin{aligned}
& \gamma_{h}=y^{2}\left(1-q^{h+1}\right)+\left(1-q^{h}\right)+y q^{h}\left(2-q^{h+1}-q^{h}\right), \\
& \lambda_{h}=y\left(1-q^{h}\right)^{2}\left(y+q^{h-1}\right)\left(1+y q^{h}\right) .
\end{aligned}
$$

The theorem is now a consequence of another lemma which gives the value of $c_{k}$.

Lemma 8.3. With $\gamma_{h}$ and $\lambda_{h}$ as in (29), we have:

$$
\begin{array}{r}
\frac{1}{(1+z)\left(1+y^{2} z\right)-\gamma_{0} z}-\frac{\lambda_{1} z^{2}}{(1+z)\left(1+y^{2} z\right)-\gamma_{1} z}-\frac{\lambda_{2} z^{2}}{(1+z)\left(1+y^{2} z\right)-\gamma_{2} z}-\cdots \\
=\sum_{k \geq 0} z^{k} \sum_{j=0}^{k} y^{k-j}(-1)^{j} \sum_{i=0}^{2 j} y^{i} q^{i(2 j-i+1) / 2} .
\end{array}
$$

Proof. After multiplying by $1-y z$, this is equivalent to

$$
\begin{aligned}
\frac{1-y z}{(1+z)\left(1+y^{2} z\right)-\gamma_{0} z}-\frac{\lambda_{1} z^{2}}{(1+z)\left(1+y^{2} z\right)-\gamma_{1} z} & -\frac{\lambda_{2} z^{2}}{(1+z)\left(1+y^{2} z\right)-\gamma_{2} z}-\cdots \\
& =\sum_{j \geq 0}(-z)^{j} \sum_{i=0}^{2 j} y^{i} q^{i(2 j-i+1) / 2} .
\end{aligned}
$$

A continued fraction expansion of the right-hand side is obtained in the work of the second and third authors [18]. More precisely, the substitution 
$(z, y, q) \mapsto\left(-y z, y q^{\frac{1}{2}}, q^{\frac{1}{2}}\right)$ in $[18$, Theorem 7.1$]$ gives

$$
\sum_{j \geq 0}(-z)^{j} \sum_{i=0}^{2 j} y^{i} q^{i(2 j-i+1) / 2}=\frac{1}{1-y z}+\frac{d_{1} z}{1-y z}+\frac{d_{2} z}{1-y z}+\frac{d_{3} z}{1-y z}+\cdots
$$

where $d_{2 h+1}=\left(1+y q^{h+1}\right)\left(y+q^{h}\right)$ and $d_{2 h}=y\left(1-q^{h}\right)^{2}$. It is immediate to check the relations

$$
\lambda_{h}=d_{2 h-1} d_{2 h}, \quad \gamma_{h}=(1+y)^{2}-d_{2 h}-d_{2 h+1}
$$

(with the convention $d_{0}=0$ ). It remains to identify the left-hand side of (30) with the right-hand side of (31). The end of the proof follows from the lemma below.

Lemma 8.4. Let $\left(\gamma_{h}\right)_{h \geq 0},\left(\lambda_{h}\right)_{h \geq 1}$ and $\left(d_{h}\right)_{h \geq 0}$ be three sequences satisfying (32) with $d_{0}=0$. Then we have:

$$
\begin{array}{r}
\frac{1-y z}{(1+z)\left(1+y^{2} z\right)-\gamma_{0} z}-\frac{\lambda_{1} z^{2}}{(1+z)\left(1+y^{2} z\right)-\gamma_{1} z}-\frac{\lambda_{2} z^{2}}{(1+z)\left(1+y^{2} z\right)-\gamma_{2} z}-\cdots \\
=\frac{1}{1-y z}+\frac{d_{1} z}{1-y z}+\frac{d_{2} z}{1-y z}+\frac{d_{3} z}{1-y z}+\cdots .
\end{array}
$$

Proof. In the left-hand side, divide the numerator and denominator of each fraction by $1-y z$. Using that

$$
\frac{(1+z)\left(1+y^{2} z\right)}{1-y z}=1-y z+\frac{z(1+y)^{2}}{1-y z}
$$

the left-hand side of $(33)$ is

$$
\frac{1}{1-y z+\left((1+y)^{2}-\gamma_{0}\right) \frac{z}{1-y z}}-\frac{\lambda_{1}\left(\frac{z}{1-y z}\right)^{2}}{1-y z+\left((1+y)^{2}-\gamma_{1}\right) \frac{z}{1-y z}}-\cdots,
$$

hence it is equal to

$$
\frac{1}{1-y z+d_{1 \frac{z}{1-y z}}}-\frac{d_{1} d_{2}\left(\frac{z}{1-y z}\right)^{2}}{1-y z+\left(d_{2}+d_{3}\right) \frac{z}{1-y z}}-\frac{d_{3} d_{4}\left(\frac{z}{1-y z}\right)^{2}}{1-y z+\left(d_{4}+d_{5}\right) \frac{z}{1-y z}}-\cdots
$$

This can be shown to be equal to the right-hand side of (33), using the combinatorics of weighted Schröder paths. A Schröder path (of length $2 n$ ) is a path from $(0,0)$ to $(2 n, 0)$ in $\mathbb{N}^{2}$ with steps $(1,1),(1,-1)$ and $(2,0)$, respectively denoted by $\nearrow, \searrow, \longrightarrow$. We set that each step $\longrightarrow$ has a weight $y$, each 
Crossings of signed permutations and $q$-Eulerian numbers of type $B 223$

step $\nearrow$ from height $h-1$ to $h$ has weight $-d_{h}$. Then by standard methods, the weighted generating function of all Schröder paths is the continued fraction in the right-hand side of (33). By counting differently, we can obtain (34). The idea is to split each Schröder path into some subpaths, by putting a splitting point each time the path arrives at even height. This way, we can see a Schröder path as an ordered sequence of:

- A subsequence $\nearrow \longrightarrow \cdots \longrightarrow \searrow$ starting at height $2 h$ whose generating function is $-d_{2 h+1} \frac{z}{1-y z}$.

- A subsequence $\searrow \longrightarrow \cdots \longrightarrow \nearrow$ starting at height $2 h$ whose generating function is $-d_{2 h} \frac{z}{1-y z}$.

- A subsequence $\nearrow \longrightarrow \cdots \longrightarrow \nearrow$ starting at height $2 h$ whose generating function is $d_{2 h+1} d_{2 h+2} \frac{z}{1-y z}$.

- A subsequence $\searrow \longrightarrow \cdots \longrightarrow \searrow$ starting at height $2 h$ whose generating function is $\frac{z}{1-y z}$.

- A step $\longrightarrow$ at height $2 h$ whose generating function is $y z$.

The rules according to which these subsequences can be put together are conveniently encoded in a continued fraction so that we obtain exactly (34). More precisely, let $F_{h}$ be the generating function of Schröder paths from height $2 h$ to $2 h$ and staying at height $\geq 2 h-1$. Note that when $h=0$, we recover the generating function of Schröder paths, because $d_{0}=0$ implies that the paths reaching height -1 have weight 0 . We have:

$$
F_{h}=\frac{1}{1-y z+\left(d_{2 h}+d_{2 h+1}\right) \frac{z}{1+y z}-d_{2 h+1} d_{2 h+2}\left(\frac{z}{1-y z}\right)^{2} F_{h+1}} .
$$

Indeed, we can see a Schröder paths from height $2 h$ to $2 h$ and staying at height $\geq 2 h-1$ as an ordered sequence of:

- steps $\longrightarrow$ at height $2 h$ whose generating function is $y z$,

- subsequences $\nearrow \longrightarrow \cdots \longrightarrow \searrow$ starting at height $2 h$ whose generating function is $-d_{2 h+1} \frac{z}{1-y z}$,

- subsequences $\searrow \longrightarrow \cdots \longrightarrow \nearrow$ starting at height $2 h$ whose generating function is $-d_{2 h} \frac{z}{1-y z}$,

- subsequences $\nearrow \longrightarrow \cdots \longrightarrow \nearrow P \searrow \longrightarrow \cdots \longrightarrow \searrow$ where $P$ is a path from height $2 h+2$ to $2 h+2$ staying above height $2 h+1$ whose generating function is $d_{2 h+1} d_{2 h+2}\left(\frac{z}{1-y z}\right)^{2} F_{h+1}$.

Hence we obtain (35). Using (35) for successive values of $h$, we obtain that $F_{0}$ is the continued fraction in (34). This completes the proof. 
Let us examine the case $q=0$ in the formula (23). We obtain:

$$
\begin{aligned}
B_{n}(y, 1,0) & =\sum_{k=0}^{n}(-1)^{k} \sum_{i=0}^{2 n-2 k} y^{i}\left(\begin{array}{c}
n \\
k+\lceil i / 2\rceil
\end{array}\right)\left(\begin{array}{c}
n \\
\lfloor i / 2\rfloor
\end{array}\right) \\
& =\sum_{i=0}^{2 n} y^{i}\left(\begin{array}{c}
n \\
\lfloor i / 2\rfloor
\end{array}\right) \sum_{k=0}^{\left\lfloor n-\frac{i}{2}\right\rfloor}(-1)^{k}\left(\begin{array}{c}
n \\
k+\lfloor i / 2\rfloor
\end{array}\right) .
\end{aligned}
$$

The alternating sum of binomial coefficients is itself a binomial coefficient, and we obtain:

$$
B_{n}(y, 1,0)=\sum_{i=1}^{2 n} y^{i}\left(\begin{array}{c}
n \\
\lfloor i / 2\rfloor
\end{array}\right)\left(\begin{array}{c}
n-1 \\
\lceil i / 2\rceil-1
\end{array}\right) .
$$

We thus obtain, as mentioned in Theorem 2.1, that the value at $q=0$ of the $q$-Eulerian numbers of type $B$ are the Narayana numbers of type $B$ :

$$
\begin{aligned}
E_{n, k}^{B}(0) & =\left[y^{2 k}\right] B_{n}(y, 1,0)+\left[y^{2 k+1}\right] B_{n}(y, 1,0) \\
& =\left(\begin{array}{l}
n \\
k
\end{array}\right)\left(\begin{array}{c}
n-1 \\
k-1
\end{array}\right)+\left(\begin{array}{c}
n \\
k
\end{array}\right)\left(\begin{array}{c}
n-1 \\
k
\end{array}\right)=\left(\begin{array}{l}
n \\
k
\end{array}\right)^{2} .
\end{aligned}
$$

Remark 1 . One can prove the identity $E_{n, k}^{B}(0)=\left(\begin{array}{l}n \\ k\end{array}\right)^{2}$ bijectively as follows. Considering $\pi \in B_{n}$ as a permutation on $[ \pm n]=\{ \pm 1, \pm 2, \ldots, \pm n\}$, define $f(\pi)$ to be the partition of $[ \pm n]$ obtained by making cycles of $\pi$ into blocks. It is not difficult to show that the map $f$ is a bijection from the set of $\pi \in B_{n}$ with $\operatorname{cr}(\pi)=0$ to the set of type $B$ noncrossing partitions of $[ \pm n]$ such that if $\lfloor$ fwex $(\pi) / 2\rfloor=k$ then $f(\pi)$ has $2 k$ nonzero blocks. It is well known that $\left(\begin{array}{l}n \\ k\end{array}\right)^{2}$ is the number of type $B$ noncrossing partitions of $[ \pm n]$ with $2 k$ nonzero blocks, see [25].

\section{Open problems}

We conclude this paper by a few open problems.

Problem 1. Since the introduction of permutation tableaux in $[28,31]$, several variants have been defined $[2,3,30]$. A nice feature of these variants is that the permutation statistics arise naturally, from a recursive construction of the tableaux via an insertion algorithm [3]. The type $B$ version of these tableaux can be defined with the condition of being conjugate-symmetric. A natural question is to check whether the insertion algorithm can be used to recover some of our results. 
Crossings of signed permutations and $q$-Eulerian numbers of type $B 225$

Problem 2. One key feature of our new $q$-Eulerian polynomials of type $B$ is their symmetry, i.e. we have $B_{n, k}^{*}(t, q)=B_{n, 2 n+1-k}^{*}(t, q)$. We prove this symmetry using the pignose diagram of a signed permutation. It would be interesting to show this symmetry using the permutation tableaux of type $B$.

Problem 3. We have defined alignments in Section 5 and showed that for a signed permutation $\pi$ with fwex $(\pi)=k$, we have $2 \operatorname{cr}(\pi)+\operatorname{al}(\pi)=n^{2}-2 n+k$. A similar identity exists for the type $A$, see Proposition 5.1, and can be shown on permutations or directly on permutation tableaux. It would be elegant to show our identity directly on the permutation tableaux of type $B$.

Problem 4. A notion that is closely related to alignments, and in some sense dual to the crossing, is the one of nesting [6]. When we introduce a parameter $p$ counting the number of crossings in permutations, there are continued fractions containing $p, q$-integers rather than the $q$-integers, see $[6,26]$. A definition of nestings in signed permutations have been given by Hamdi [16]. It would be interesting to check if our results can be generalized to take into account these nestings.

Problem 5. In the last section, we have obtained a formula for $B_{n}(y, 1, q)$. We can ask if there is a more general formula for $B_{n}(y, t, q)$, but it seems that the present methods do not generalize in this case.

Problem 6. Recently Kim and Stanton [20] gave a combinatorial proof of the formula (3) for $B_{n}(y, 0, q)$, which is a generating function for type $A$ permutation tableaux. It is worth asking whether this combinatorial approach can be generalized for $B_{n}(y, 1, q)$ and possibly $B_{n}(y, t, q)$.

\section{Acknowledgements}

We thank Philippe Nadeau and Lauren Williams for constructive discussions during the elaboration of this work.

\section{References}

[1] R. M. Adin, F. Brenti and Y. Roichman (2001). Descent numbers and major indices for the hyperoctahedral group. Adv. in Appl. Math. 27(23) 210-224. Special issue in honor of Dominique Foata's 65th birthday (Philadelphia, PA, 2000). MR1868962

[2] J.-C. Aval, A. Boussicault and S. Dasse-Hartaut (2011). Dyck tableaux. Theoret. Comput. Sci., in press. 
[3] J.-C. Aval, A. Boussicault and P. Nadeau (2011). Tree-like tableaux. In: Proc. of FPSAC 2011, Discrete Math. Theor. Comput. Sci. Proc., 63-74. MR2820698

[4] A. Björner and F. Brenti (2005). Combinatorics of Coxeter Groups. Springer. MR2133266

[5] L. Comtet (1974). Advanced Combinatorics. Reidel Publishing Company. MR0460128

[6] S. Corteel (2007). Crossings and alignments of permutations. Adv. in Appl. Math. 38(2) 149-163. MR2290808

[7] S. Corteel, M. Josuat-Vergès and L. K. Williams (2011). Matrix Ansatz, orthogonal polynomials and permutations. Adv. in Appl. Math. 46 209225. MR2794022

[8] S. Corteel, M. Josuat-Vergès, T. Prellberg and M. Rubey (2009). Matrix Ansatz, lattice paths and rook placements. In: Proceedings of FPSAC 2009, Discrete Math. Theor. Comput. Sci. Proc., 313-324. MR2721522

[9] S. Corteel and J. S. Kim (2011). Combinatorics on permutation tableaux of type $A$ and type B. European J. Combin. 32(4) 563-579. MR2780856

[10] S. Corteel and L. K. Williams (2007). A Markov chain on permutations which projects to the asymmetric exclusion process. Int. Math. Res. Not. article ID mm055.

[11] S. Corteel and P. Nadeau (2009). Bijections for permutation tableaux. European J. Combin. 30 295-300. MR2460235

[12] P. Flajolet (1982). Combinatorial aspects of continued fractions. Discrete Math. 41 145-153. MR0676874

[13] D. Foata and G. Han (2009). Signed words and permutations, V; a sextuple distribution. Ramanujan J. 19 (1) 29-52. MR2501235

[14] D. Foata and D. Zeilberger (1990). Denert's permutation statistic is indeed Euler-Mahonian. Stud. Appl. Math. 83(1) 31-59. MR1061147

[15] J. Françon and X. G. Viennot (1979). Permutations selon leurs pics, creux, doubles montées et double descentes, nombres d'Euler et nombres de Genocchi. Discrete Math. 28 21-35. MR0542933

[16] A. Hamdi (2011). Symmetric distribution of crossings and nestings in permutations of type B. Electr. J. Comb. 18(1) Article P200. MR2853057 
Crossings of signed permutations and $q$-Eulerian numbers of type $B 227$

[17] M. Josuat-Vergès (2011). Rook placements in Young diagrams and permutation enumeration. Adv. in Appl. Math. 47 1-22. MR2799608

[18] M. Josuat-Vergès and J. S. Kim (2013). Touchard-Riordan formulas, T-fractions, and Jacobi's triple product identity. Ramanujan J. 30(3) 341-378. MR3033681

[19] M. Josuat-Vergès and M. Rubey (2011). Crossings, Motzkin paths, and moments. Discrete Math. 311 2064-2078. MR2819649

[20] J. S. Kim and D. Stanton (2013). Moments of Askey Wilson polynomials. arxiv:1207.3446.

[21] T. Lam and L. K. Williams (2008). Total positivity for cominuscule Grassmannians. New York J. of Math. 14 53-99. MR2383586

[22] P. Leroux and X. G. Viennot (1986). Combinatorial resolution of systems of differential equations, I. Ordinary differential equations. In Combinatoire énumérative, G. Labelle and P. Leroux, eds, Lecture Notes in Maths., Vol. 1234, Springer-Verlag, Berlin, 1986, pp. 210-245. MR0927767

[23] A. de Médicis and X. G. Viennot (1994). Moments des q-polynômes de Laguerre et la bijection de Foata-Zeilberger. Adv. in Appl. Math. 15(3) 262-304. MR1288802

[24] A. Postnikov (2006). Total positivity, Grassmannians, and networks. Preprint. arxiv:0609764.

[25] V. Reiner (1997). Non-crossing partitions for classical reflection groups. Discrete Math. 177 195-222. MR1483446

[26] H. Shin and J. Zeng (2012). The symmetric and unimodal expansion of Eulerian polynomials via continued fractions. European J. Combin. 33(2) 111-127. MR2854634

[27] R. P. Stanley (2011). Enumerative Combinatorics. Vol. 1, 2nd ed., (version of 15 July 2011). To be published in Cambridge University Press.

[28] E. Steingrímsson and L. K. Williams (2007). Permutation tableaux and permutation patterns. J. Combin. Theory Ser. A 114 211-234. MR2293088

[29] X. G. Viennot (1984). Une théorie combinatoire des polynômes orthogonaux. Lecture notes, UQÀM, Montréal.

[30] X. G. Viennot (2008). Alternative tableaux and partially asymmetric exclusion process. Talk in Isaac Newton institute. 
[31] L. K. Williams (2005). Enumeration of totally positive Grassmann cells. Adv. Math. 190 319-342. MR2102660

Sylvie CoRTeEl

LIAFA, CNRS AND Université PARIS-Diderot

Case 7014, 75205 Paris Cedex 13

FRANCE

E-mail address: corteel@liafa.univ-paris-diderot.fr

Matthieu Josuat-Vergès

Institut Gaspard Monge, CNRS and Université de Marne-la-Vallée

5 Boulevard Descartes, 77454 Champs-sur-Marne

MARNe-la-VALlÉE CEDEX

FRANCE

E-mail address: matthieu.josuat-verges@univ-mlv.fr

JANG SOO KIM

School of Mathematics, University of Minnesota

Minneapolis, Minnesota 55455

USA

E-mail address: kimjs@math. umn.edu

Received March 1, 2012 